\title{
Unification of Dynamic Density Functional Theory for Colloidal Fluids to Include Inertia and Hydrodynamic Interactions: Derivation and Numerical Experiments
}

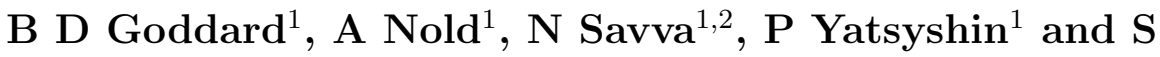 \\ Kalliadasis $^{1}$ \\ ${ }^{1}$ Department of Chemical Engineering, Imperial College London, South Kensington \\ Campus, London SW7 2AZ, United Kingdom \\ ${ }^{2}$ Cardiff School of Mathematics, Cardiff University, Cardiff, CF24 4AG, United \\ Kingdom \\ E-mail: b.goddard@imperial.ac.uk, s.kalliadasis@imperial.ac.uk
}

\begin{abstract}
Starting from the Kramers equation for the phase-space dynamics of the $N$-body probability distribution, we derive a dynamical density functional theory (DDFT) for colloidal fluids including the effects of inertia and hydrodynamic interactions (HI). We compare the resulting theory to extensive Langevin dynamics simulations for both hard rod systems and three-dimensional hard sphere systems with radially-symmetric external potentials. As well as demonstrating the accuracy of the new DDFT, by comparing with previous DDFTs which neglect inertia, HI, or both, we also scrutinise the significance of including these effects. Close to local equilibrium we derive a continuum equation from the microscopic dynamics which is a generalized Navier-Stokes-like equation with additional non-local terms governing the effects of HI. In the overdamped limit we recover analogues of existing configuration-space DDFTs but with a novel diffusion tensor.
\end{abstract}

PACS numbers: 05.20.Jj, 47.10.A-, 47.57.J-, 82.70.Dd

\section{Introduction}

In fluid dynamics it is often sufficient to model the individual fluid particles as a continuum, for example by employing the Navier-Stokes equation. However, for colloidal fluids where mesoscopic particles (typically of size $1 \mathrm{~nm}-1 \mu \mathrm{m}$ ) are suspended in a bath of many more, much smaller and much lighter particles, one is interested in the dynamics on length scales comparable to the size of the colloidal particles [1]. In this case, a continuum, macroscopic formalism is insufficient.

Since the experimental observations of the Brownian motion of pollen particles in water in the 19th Century [2], the study of classical fluids (systems of particles at sufficiently high temperature so that quantum effects can be neglected), such as colloidal 
systems, has been fundamental not only to the development of statistical mechanics starting from the work of Einstein [3], Langevin [4] and Smoluchowski [5], but also to many other fields in physics, chemistry and engineering. Examples include the evolution of microscopy over the last century $[6,7,8]$, recent advances in biophysical research [9] and the rapidly-growing field of microfluidics [10, 11, 12]. Such colloidal systems are important model ones for both theoretical and experimental scrutiny. Many of the forces governing their structure and behaviour govern also the structure and behaviour of molecular matter, whilst the sufficiently large physical size of colloidal particles enables them to be accessed experimentally.

The full dynamics of the system are described by the deterministic Newtonian dynamics of the positions and momenta of both the colloidal and bath particles. However, since the bath particles are much lighter than the colloidal particles, their typical velocities are much higher than those of the colloidal particles. Hence, if one is interested in only the dynamics of the colloidal particles, the multi-scale nature of the problem enables one to average out the rapid fluctuations in the positions and momenta of the bath particles. Such a description is valid on timescales $t \gg \tau_{b}$, where $\tau_{b}$ is the typical time between collisions of the bath particles. In this coarse-grained model, only the positions and momenta of the colloid particles are treated explicitly. The need for such an approximation can be seen from the fact that a colloidal particle of diameter $1 \mu \mathrm{m}$ occupies the same volume as the order of $10^{10}$ water molecules. A typical collision timescale for the water molecules is $10^{-15} \mathrm{~s}$, whilst a typical colloid particle will take about 1s to diffuse a distance comparable to its diameter. Hence, modelling the full system is computationally intractable due both to the large number of particles and the wide range of timescales which must be considered.

A further complication of colloidal systems compared to molecular fluids arises from the interaction of the colloidal and bath particles. The motion of the colloidal particles causes flows in the bath, which in turn cause forces on the colloidal particles. These hydrodynamic interactions (HI), along with thermally-induced collisions of bath particles with colloidal particles, are modelled via stochastic Langevin equations for the particle positions and momenta, or the corresponding Fokker-Planck equations for the probability distributions. However, for large numbers of particles, these equations become computationally prohibitive and a reduced model is required.

One technique for obtaining such reduced models is dynamical density functional theory (DDFT). This is a statistical mechanics approach in which one aims to reduce the full dynamics to closed equations for the dynamics of one-body quantities, such as the one-body position distribution $\rho(\mathbf{r}, t)$. A typical DDFT is a continuity equation for the density $\partial_{t} \rho(\mathbf{r}, t)+\boldsymbol{\nabla}_{\mathbf{r}} \cdot \mathbf{J}([\rho], \mathbf{r}, t)=0$, where $\mathbf{J}$ is a functional of the density. The main computational advantage of such DDFTs over the full $N$-body dynamics is that of dimensionality. The full description of $N$ particles in $d$ dimensions requires the study of $2 d N$ variables ( $d N$ positions and $d N$ momenta), whilst DDFTs are PDEs in only the $d$ spatial variables and one time variable. This is independent of the number of particles $N$, allowing arbitrarily large numbers of particles to be studied 
for constant computational cost. Whilst it has been shown rigorously that the full $N$ body distribution is a functional of the one-body distribution [13], the functional itself is unknown. For computations this functional, or a good approximation to it, needs to be known explicitly. Due to the large body of work and success of equilibrium density functional theory (DFT) (see [14, 15] for early work and [16, 17] for recent reviews), this functional is usually based upon the free energy functional of a related equilibrium system. This approach also ensures that the DDFT reduces to the correct static DFT at equilibrium. In this sense DDFT is a direct generalisation of DFT.

Beyond the separation of colloidal and bath dynamics described above, there are several additional natural timescales associated with the colloidal particle dynamics [18], of which we consider only $\tau_{B}$, the typical relaxation time of the colloidal particle momenta due to friction with the bath. A typical value is $\tau_{B}=10^{-7} \mathrm{~s}$ [19]. For times $t \gg \tau_{B}$, the momenta of the colloidal particles are at equilibria, and interest lies in the dynamics of their positions.

DDFT formulations for the colloidal position density were first obtained phenomenologically by Evans [14] and Dietrich et al. [20]. There has since been an effort to more rigorously derive such a result from the Smoluchowski equation: in this way Marconi and Tarazona [21, 22] derived a DDFT for pairwise potentials. $\partial_{t} \rho(\mathbf{r}, t)=\boldsymbol{\nabla}_{\mathbf{r}} \cdot\left[\rho(\mathbf{r}, t) \boldsymbol{\nabla}_{\mathbf{r}} \delta F[\rho(\mathbf{r}, t)] / \delta \rho(\mathbf{r}, t)\right]$, where $F[\rho]$ is the free energy functional for an equilibrium system with the same one-particle density. This is commonly called the adiabatic approximation. Later Archer and Evans [23] obtained the analogous result for general $N$-body interaction potentials, including the incorporation of a mobility constant. Español and Löwen [24] derived an analogous result using projection operator techniques.

All of these results ignore HI. In contrast, Rex and Löwen [25] derived a DDFT from the Smoluchowski equation with a two-body diffusion tensor and interparticle potential to include HI. Attempts have also been made to model hydrodynamic interactions via a density-dependent friction coefficient [26, 27].

Despite the typical timescale separation discussed earlier, there are a wide range of applications in which the momenta of the particles is important. Examples include wetting phenomena [28, 29, 30], aerosol deposition and cloud formation $[31,32,33,34,35]$, the transport and coagulation of nanoparticles in pulsatile flow, such as in the cardiovascular system and oscillatory flow mixing [36]. In many of these applications HI also play a crucial role, strongly affecting flow patterns and clustering effects.

In order to incorporate the effect of inertia into DDFTs, two different approaches have been considered. The first, introduced by Marconi, Tarazona and coworkers [37, 38, 39, 40, 41], uses a multiple timescale approach. The second approach by Archer [42] takes momentum moments of the Kramers equation, giving a DDFT coupled to an evolution equation for the one-body velocity distribution. This is the approach we will take here. As with the majority of the configuration-space DDFTs mentioned above, none of these previous approaches includes HI. A first stab at the problem of deriving 
a general DDFT was the recent study in [43]. In the high friction limit, and neglecting HI, this reduces to the configuration space DDFT of Marconi and Tarazona as was shown in the recent study in [44] which also includes a schematic synopsis of many previous DDFTs. Under additional assumptions on the form of the friction tensor, it also recovers the result of Rex and Löwen, including HI, but in general results in a novel diffusion tensor. Here we build on this initial work, outlining in detail the methodologies, both existing (e.g. Grad's moment approach) and novel, required for the derivation of a general DDFT, discussing the various approximations as well as extending the numerical validation and experiments to time-dependent external potentials for both 3D hard spheres and 1D hard rods. In addition, we demonstrate the connections between our DDFT and the Navier-Stokes equation (close to local equilibrium) and existing, overdamped DDFTs (in the high-friction limit).

In the following we will derive a DDFT from the Kramers equation with a general friction tensor and inter-particle potential. We will show that, under suitable assumptions, we obtain many existing DDFTs as special cases. In Section 2 we discuss the standard underlying $N$-body equations of motion and compute explicitly the first two equations in an infinite hierarchy for the dynamics of the momentum moments of the density. In Section 3 we make three approximations, allowing us to derive our DDFT, which we then verify numerically in Section 4. In Section 5 we assume in addition that the system is close to local equilibrium and derive a Navier-Stokes-like integrodifferential equation. In Section 6 we show that, in the high friction regime we recover a position-space DDFT, which under additional assumptions reduces to that of Rex and Löwen [25]. Finally, concluding remarks are offered in Section 7 where we also discuss some of the many open problems in this rapidly-emerging field.

\section{Equations of Motion}

We consider a system with $N$ identical, spherically symmetric colloidal particles, in a bath of many more, much smaller and lighter solvent particles. As described in the Introduction, it is assumed that the dynamics of the bath particles relaxes on a much shorter time-scale than that of the colloidal particles, and hence the intrabath interactions may be treated via coarse-graining. Interest then lies in the positions $\mathbf{r}^{N}=\left\{\mathbf{r}_{1}, \ldots, \mathbf{r}_{N}\right\}$ and momenta $\mathbf{p}^{N}=\left\{\mathbf{p}_{1}, \ldots, \mathbf{p}_{N}\right\}$ of the colloidal particles.

The underlying dynamics of the colloidal particles are described by the Langevin equations

$$
\dot{\mathbf{r}}_{i}=\frac{\mathbf{p}_{i}}{m}, \quad \dot{\mathbf{p}}_{i}=-\nabla_{\mathbf{r}_{i}} V\left(\mathbf{r}^{N}, t\right)-\sum_{j=1}^{N} \boldsymbol{\Gamma}_{i j}\left(\mathbf{r}^{N}\right) \mathbf{p}_{j}+\sum_{j=1}^{N} \mathbf{A}_{i j} \mathbf{f}_{j}(t)
$$

where $\mathbf{f}_{i}(t)=\left(\xi_{i}^{x}(t), \xi_{i}^{y}(t), \xi_{i}^{z}(t)\right)^{T}$ is a Gaussian white noise term with $\left\langle\xi_{i}^{a}(t)\right\rangle=0$ and $\left\langle\xi_{i}^{a}(t) \xi_{j}^{b}\left(t^{\prime}\right)\right\rangle=2 \delta_{i j} \delta^{a b} \delta\left(t-t^{\prime}\right)$. Along with the gradient of the potential $V$, there are two additional sets of forces due to the bath. Firstly, the motion of the colloidal particles causes fluid flows in the bath, which in turn cause forces on the colloidal particles. 
This coupling of momenta and forces, already referred to as HI, is governed by $\boldsymbol{\Gamma}$, the $3 N \times 3 N$ positive definite friction tensor $\boldsymbol{\Gamma}\left(\mathbf{r}^{N}\right)=\left(\boldsymbol{\Gamma}_{i j}\left(\mathbf{r}^{N}\right)\right)$ formed from the $3 \times 3$ matrices $\boldsymbol{\Gamma}_{i j}$. Secondly, forces are generated by random collisions of the bath particles with the colloidal particles. The strength of these forces is given by $\mathbf{A}$, which is coupled to $\boldsymbol{\Gamma}$ through the generalised fluctuation-dissipation relation $\mathbf{A}=\sqrt{m k_{B} T \boldsymbol{\Gamma}}$ [45]. Here $m$ is the particle mass, $k_{B}$ is Boltzmann's constant and $T$ is the temperature, which we assume to be constant since the bath acts also as a heat bath.

The corresponding Fokker-Planck equation for the evolution of the phase space distribution function, known as the Kramers equation (see e.g. [46]), is

$$
\begin{aligned}
\partial_{t} f^{(N)}\left(\mathbf{r}^{N}, \mathbf{p}^{N}, t\right)+\frac{1}{m} \sum_{i=1}^{N} \mathbf{p}_{i} \cdot \nabla_{\mathbf{r}_{i}} f^{(N)}\left(\mathbf{r}^{N}, \mathbf{p}^{N}, t\right) \\
-\sum_{i=1}^{N} \boldsymbol{\nabla}_{\mathbf{r}_{i}} V\left(\mathbf{r}^{N}, t\right) \cdot \boldsymbol{\nabla}_{\mathbf{p}_{i}} f^{(N)}\left(\mathbf{r}^{N}, \mathbf{p}^{N}, t\right) \\
=\sum_{i, j=1}^{N} \boldsymbol{\nabla}_{\mathbf{p}_{i}} \cdot\left[\boldsymbol{\Gamma}_{i j}\left(\mathbf{r}^{N}\right)\left(\mathbf{p}_{j}+m k_{B} T \boldsymbol{\nabla}_{\mathbf{p}_{j}}\right) f^{(N)}\left(\mathbf{r}^{N}, \mathbf{p}^{N}, t\right)\right] .
\end{aligned}
$$

Here, $f^{(N)}\left(\mathbf{r}^{N}, \mathbf{p}^{N}, t\right)$ is the probability of finding each particle $j$ at position $\mathbf{r}_{j}$ with momentum $\mathbf{p}_{j}$ at time $t$. We note that in the above we have used the notation $\mathbf{r}^{N}=\mathbf{r}_{1}, \ldots, \mathbf{r}_{N}$ and analogously for $\mathbf{p}^{N}$. In the following we generalise this notation to $\mathbf{r}^{n}=\mathbf{r}_{1}, \ldots, \mathbf{r}_{n}$ and $\mathrm{d} \mathbf{r}^{N-n}=\mathrm{d} \mathbf{r}_{n+1} \ldots \mathrm{d} \mathbf{r}_{N}$, with corresponding expressions in $\mathbf{p}$.

Since the solution of the Langevin equations (1) requires finding the square root of $\boldsymbol{\Gamma}$, a standard timestepping algorithm will require $O\left(N^{3}\right)$ operations at each timestep, and quickly becomes computationally intractable. For large $N$, solving Kramers equation (2) is no simpler as it is a high-dimensional partial differential equation (PDE), which must be solved via Monte-Carlo methods, with the same difficulties as the solution of (1). One way to overcome this limitation is to ignore HI by setting $\boldsymbol{\Gamma}=\gamma \mathbf{1}$ for some constant $\gamma$. Whilst this reduces the computational scaling to $O(N)$, it also ignores many important physical effects. We now describe a reduced, computationally tractable model, which retains the full HI.

A standard approach for both classical (e.g. the Navier-Stokes equations) and quantum (e.g. density functional theory) systems is to average over the degrees of freedom of all but a few particles, leading to a lower-dimensional problem. In both the classical [13] and quantum [47] cases it is known rigourously that the full $N$ particle probability distribution is a functional of the one-body position distribution $\rho\left(\mathbf{r}_{1}, t\right)=\int \mathrm{d} \mathbf{r}^{N-1} \mathrm{~d} \mathbf{p}^{N} f^{(N)}\left(\mathbf{r}^{N}, \mathbf{p}^{N}, t\right)$. Hence, in principle, the solution of (2) can be determined by solving a $(3+1)$-dimensional PDE. The difficulty lies in the fact that the functional relating $\rho\left(\mathbf{r}_{1}, t\right)$ to $f^{(N)}\left(\mathbf{r}^{N}, \mathbf{p}^{N}, t\right)$ is unknown. In fact, the equilibrium functional relating $\rho(\mathbf{r}, t)$ to $f^{(N)}\left(\mathbf{r}^{N}, \mathbf{p}^{N}\right)$ is also unknown for all but the simplest onedimensional (1D) cases [48]. The challenge is therefore to find suitable approximate closures for the dynamics of $\rho\left(\mathbf{r}_{1}, t\right)$, i.e. to derive a DDFT.

We now make our first approximation, namely that the potential may be written 
as a linear combination of $n$-particle interactions:

$$
V\left(\mathbf{r}^{N}, t\right)=\sum_{i=1}^{N} V_{1}\left(\mathbf{r}_{i}, t\right)+\sum_{n=2}^{N} \frac{1}{n !} \sum_{i_{1} \neq \ldots \neq i_{n}=1}^{N} V_{n}\left(\mathbf{r}_{i_{1}}, \ldots, \mathbf{r}_{i_{n}}\right),
$$

where we have assumed that the inter-particle potentials depend on time only through the time-dependent particle positions, whilst the external potential may be explicitly time-dependent. For ease of notation, we write the friction tensor as

$$
\boldsymbol{\Gamma}_{i j}=\gamma \delta_{i j} \mathbf{1}+\gamma \tilde{\boldsymbol{\Gamma}}_{i j},
$$

where $\gamma$ is the friction coefficient for a single, isolated particle and the $\tilde{\boldsymbol{\Gamma}}_{i j}$ are the HI tensors. Furthermore, when $\gamma=0$, this reduces to the Liouville equation, i.e. the dynamics in the absence of a bath. In this way, the discussions below also apply to molecular fluids, but it is more difficult, except in very special cases, to justify the local-equilibrium approximation discussed in Section 3.2.

We now define the set of reduced phase space distribution functions

$$
f^{(n)}\left(\mathbf{r}^{n}, \mathbf{p}^{n}, t\right)=\frac{N !}{(N-n) !} \int \mathrm{d} \mathbf{r}^{N-n} \mathrm{~d} \mathbf{p}^{N-n} f^{(N)}\left(\mathbf{r}^{N}, \mathbf{p}^{N}, t\right),
$$

and corresponding configuration space distributions

$$
\rho^{(n)}\left(\mathbf{r}^{n}, t\right)=\int \mathrm{d} \mathbf{p}^{n} f^{(n)}\left(\mathbf{r}^{n}, \mathbf{p}^{n}, t\right),
$$

where for ease of notation we denote $\rho^{(1)}$ by $\rho$. Our strategy is to determine the momentum moments of the Kramers equation (2), giving an infinite hierarchy of equations which must then be truncated. We begin by integrating (2) over all but one particle position $\left(N \int \mathrm{d} \mathbf{r}^{N-1} \mathrm{~d} \mathbf{p}^{N}(2)\right)$, giving a continuity equation

$$
\partial_{t} \rho(\mathbf{r}, t)+\nabla_{\mathbf{r}} \cdot \mathbf{j}=0
$$

where, as before, $\rho$ is the one-body density and $\mathbf{j}$ is the current $\mathbf{j}(\mathbf{r}, t)=$ $\int \mathrm{d} \mathbf{p} \frac{\mathbf{p}}{m} f^{(1)}(\mathbf{r}, \mathbf{p}, t)$.

At this stage, the HI have no effect (as they conserve mass). However, they enter the equation for the current, which we obtain by multiplying (2) by $N \mathbf{p}_{1} / m$ and again integrating over all but $\mathbf{r}_{1}$, giving (after some integration by parts and use of the divergence theorem in the HI term)

$$
\begin{aligned}
\partial_{t} \mathbf{j}\left(\mathbf{r}_{1}, t\right)+\nabla_{\mathbf{r}_{1}} & \cdot \int \mathrm{d} \mathbf{p}_{1} \frac{\mathbf{p}_{1} \otimes \mathbf{p}_{1}}{m^{2}} f^{(1)}\left(\mathbf{r}_{1}, \mathbf{p}_{1}, t\right)+\frac{1}{m} \rho\left(\mathbf{r}_{1}, t\right) \boldsymbol{\nabla}_{\mathbf{r}_{1}} V_{1}\left(\mathbf{r}_{1}, t\right) \\
& +\frac{1}{m} \sum_{n=2}^{N} \int \mathrm{d} \mathbf{r}^{n-1} \nabla_{\mathbf{r}_{1}} V_{n}\left(\mathbf{r}^{n}\right) \rho^{(n)}\left(\mathbf{r}^{n}, t\right)+\gamma \mathbf{j}\left(\mathbf{r}_{1}, t\right) \\
& +\frac{\gamma N}{m} \sum_{j=1}^{N} \int \mathrm{d} \mathbf{r}^{N-1} \mathrm{~d} \mathbf{p}^{N} \tilde{\boldsymbol{\Gamma}}_{1 j}\left(\mathbf{r}^{N}\right) \mathbf{p}_{j} f^{(N)}\left(\mathbf{r}^{N}, \mathbf{p}^{N}, t\right)=0,
\end{aligned}
$$

where $\otimes$ denotes the dyadic or tensor product. 
Whilst we could compute higher momentum moments of (2), we instead now aim to close the hierarchy and derive a DDFT involving only functionals of the density. The three terms in (6) which need to be approximated are (i) the term involving the dyadic product, which is reminiscent of the divergence of a kinetic pressure tensor, (ii) the many-body potential terms, and (iii) the HI terms.

In the next section, we will first remove the dependence on $\rho^{(n)}$ of the terms containing the potential via the widely used adiabatic approximation originally suggested in [21]. We then consider the dyadic product term by decomposing $f^{(1)}$ into a sum of local equilibrium and non-equilibrium terms. Finally, we discuss how to deal with the $f^{(n)}$ in the HI terms, with particular emphasis on two-body interactions. This will allow us to derive a general DDFT containing both inertia and HI. By setting $\boldsymbol{\Gamma}=\gamma \mathbf{1}$ we recover the DDFT derived by Archer [42], which neglects HI. In the large- $\gamma$ limit, we obtain an analogous DDFT to that of Rex and Löwen [25], but with a modified HI term. See [44]. This in turn, by neglecting HI, recovers the original DDFT of Marconi and Tarazona [21]. The passage to the overdamped limit was treated rigorously in [44].

\section{Derivation of the DDFT}

\subsection{The Adiabatic Approximation}

For non-equilibrium systems, little is known about appropriate choices of functionals required to close the hierarchy discussed above. However, in equilibrium systems, these functionals have received much attention and are well-understood. For example, hard sphere systems are well-approximated by fundamental measure theory (FMT) $[49,50,51,52]$ whilst mean field theory $[53,54]$ becomes exact for soft interactions at high densities [55]. As such, Marconi and Tarazona [21, 22] suggested approximating the higher-body correlations by those of an equilibrium fluid with the same density $\rho$. This is known as the adiabatic approximation.

To proceed, we consider the Helmholtz free energy functional $\mathcal{F}[\rho]=$ $k_{B} T \int \mathrm{d} \mathbf{r} \rho(\mathbf{r}, t)\left[\ln \left(\Lambda^{3} \rho(\mathbf{r}, t)\right)-1\right]+\mathcal{F}_{\text {exc }}[\rho]+\int \mathrm{d} \mathbf{r} \rho(\mathbf{r}, t) V_{1}(\mathbf{r}, t)$, which is minimized at equilibrium. Here $\Lambda$ is the de Broglie wavelength, which will turn out to be irrelevant. $\mathcal{F}$ contains an ideal gas term, a term which depends on the external potential, and an excess over ideal gas term $\mathcal{F}_{\text {exc }}[\rho]$, which is generally unknown but by definition satisfies the equilibrium sum rule

$$
\rho\left(\mathbf{r}_{1}, t\right) \boldsymbol{\nabla}_{\mathbf{r}_{1}} \frac{\delta \mathcal{F}_{\mathrm{ex}}[\rho]}{\delta \rho}=\sum_{n=2}^{N} \int \mathrm{d} \mathbf{r}_{2} \ldots \mathrm{d} \mathbf{r}_{n} \boldsymbol{\nabla}_{\mathbf{r}_{1}} V_{n}\left(\mathbf{r}^{n}\right) \rho^{(n)}\left(\mathbf{r}^{n}, t\right) .
$$

We now assume that the $n$-body densities in the true non-equilibrium system are well-approximated by the $n$-body densities in the corresponding equilibrium system with the same density, i.e. that (7) also holds out of equilibrium. Using the definition of $\mathcal{F}$ along with equations (6) and (7) gives

$\partial_{t} \mathbf{j}\left(\mathbf{r}_{1}, t\right)+\mathbf{A}\left(\mathbf{r}_{1}, t\right)+\frac{1}{m} \rho\left(\mathbf{r}_{1}, t\right) \boldsymbol{\nabla}_{\mathbf{r}_{1}} \frac{\delta \mathcal{F}[\rho]}{\delta \rho}$ 


$$
+\gamma \mathbf{j}\left(\mathbf{r}_{1}, t\right)+\frac{\gamma N}{m} \sum_{j=1}^{N} \int \mathrm{d} \mathbf{r}^{N-1} \mathrm{~d} \mathbf{p}^{N} \tilde{\boldsymbol{\Gamma}}_{1 j}\left(\mathbf{r}^{N}\right) \mathbf{p}_{j} f^{(N)}\left(\mathbf{r}^{N}, \mathbf{p}^{N}, t\right)=0,
$$

where we have defined a kinetic pressure term

$$
\mathbf{A}(\mathbf{r}, t):=\nabla_{\mathbf{r}} \cdot \int \mathrm{d} \mathbf{p}\left(\frac{\mathbf{p} \otimes \mathbf{p}}{m^{2}}-\frac{k_{B} T}{m} \mathbf{1}\right) f^{(1)}(\mathbf{r}, \mathbf{p}, t) .
$$

Note in particular that $\mathbf{A}$ is zero at equilibrium, when $f^{(1)}$ is a Maxwell-Boltzmann distribution. Furthermore, the kernel of the integral in (9) is now orthogonal to 1 and $\mathbf{p}$ in the Maxwell-Boltzmann weighted inner product; see section 3.2. We now discuss how to treat $\mathbf{A}$ away from equilibrium.

\subsection{Expansion of the one-body distribution}

One common approximation in statistical mechanics is to assume that the one-body distribution is at local equilibrium, i.e. that the system may be divided into cells sufficiently small so that the thermodynamic quantities such as density, momentum and temperature can be assumed to be constant on each cell, but also sufficiently large to be treated as macroscopic thermodynamic subsystems. [56]. For a general one-body distribution, these quantities correspond to moments of the momentum. Denoting $\int \mathrm{d} \mathbf{p} f^{(1)}(\mathbf{r}, \mathbf{p}, t)=\rho(\mathbf{r}, t), \int \operatorname{d} \mathbf{p p} f^{(1)}(\mathbf{r}, \mathbf{p}, t)=\rho(\mathbf{r}, t) m \mathbf{v}\left(\mathbf{r}_{1}, t\right)$, and $\int \mathrm{d} \mathbf{p} \mid \mathbf{p}-$ $\left.m \mathbf{v}(\mathbf{r}, t)\right|^{2} f^{(1)}(\mathbf{r}, \mathbf{p}, t)=\rho(\mathbf{r}, t) m k_{B} T$, we have a local-equilibrium approximation to the true distribution

$$
f_{\mathrm{le}}^{(1)}(\mathbf{r}, \mathbf{p}, t)=\frac{\rho(\mathbf{r}, t)}{\left(2 \pi m k_{B} T\right)^{3 / 2}} \exp \left(-\frac{|\mathbf{p}-m \mathbf{v}(\mathbf{r}, t)|^{2}}{2 m k_{B} T}\right) .
$$

Here, we go beyond local equilibrium by expanding $f^{(1)}(\mathbf{r}, \mathbf{p}, t)=f_{\mathrm{le}}^{(1)}(\mathbf{r}, \mathbf{p}, t)+$ $f_{\text {neq }}^{(1)}(\mathbf{r}, \mathbf{p}, t)$, where the local equilibrium and non-equilibrium parts satisfy

$$
\begin{aligned}
& \int \mathrm{d} \mathbf{p} f_{\mathrm{le}}^{(1)}(\mathbf{r}, \mathbf{p}, t)=\rho(\mathbf{r}, t), \\
& \int \mathrm{d} \mathbf{p} f_{\text {neq }}^{(1)}(\mathbf{r}, \mathbf{p}, t)=0, \\
& \int \mathrm{d} \mathbf{p} \mathbf{p} f_{\mathrm{le}}^{(1)}(\mathbf{r}, \mathbf{p}, t)=\rho(\mathbf{r}, t) m \mathbf{v}(\mathbf{r}, t), \\
& \int \mathrm{d} \mathbf{p} \mathbf{p} f_{\text {neq }}^{(1)}(\mathbf{r}, \mathbf{p}, t)=0 \\
& \int \mathrm{d} \mathbf{p}|\mathbf{p}-m \mathbf{v}(\mathbf{r}, t)|^{2} f_{\mathrm{le}}^{(1)}(\mathbf{r}, \mathbf{p}, t)=\rho(\mathbf{r}, t) m k_{B} T, \\
& \int \mathrm{d} \mathbf{p}|\mathbf{p}-m \mathbf{v}(\mathbf{r}, t)|^{2} f_{\text {neq }}^{(1)}(\mathbf{r}, \mathbf{p}, t)=0 .
\end{aligned}
$$

Note that an analogous partition of $f$ is used in Lattice-Boltzmann theory, where the integral restrictions are replaced by sums due to the discreteness of the LatticeBoltzmann model. See e.g. [57].

Physically, as seen above, $f_{\text {le }}^{(1)}$ determines local quantities, such as density, velocity and temperature. In contrast, $f_{\text {neq }}^{(1)}$ determines transport processes such as 
the pressure tensor and heat flux. For example, the heat flux is given by $\mathbf{q}(\mathbf{r}, t)=$ $\frac{1}{2} \int \mathrm{d} \mathbf{p}|\mathbf{w}|^{2} \mathbf{w} f^{(1)}(\mathbf{r}, \mathbf{p}, t)=\frac{1}{2} \int \mathrm{d} \mathbf{p}|\mathbf{w}|^{2} \mathbf{w} f_{\text {neq }}^{(1)}(\mathbf{r}, \mathbf{p}, t)$, where $\mathbf{w}=\mathbf{p}-m \mathbf{v}(\mathbf{r}, t)[58,(3.15)]$. The contribution from $f_{\text {le }}^{(1)}$ vanishes due to symmetry of the integral. Whilst higher moments of $\mathbf{p}$ may be treated theoretically, for practical purposes they are of less interest as they are rarely investigated experimentally,

Returning for a moment to (8), it is trivial to see from (13) and (15) that $\mathbf{j}(\mathbf{r}, t)=\mathbf{j}_{\mathrm{le}}(\mathbf{r}, t)=\rho(\mathbf{r}, t) \mathbf{v}(\mathbf{r}, t)$ and using the definition of $\mathbf{A}(9)$,

$$
\begin{aligned}
\mathbf{A}(\mathbf{r}, t)=\nabla_{\mathbf{r}} & \cdot[\rho(\mathbf{r}, t) \mathbf{v}(\mathbf{r}, t) \otimes \mathbf{v}(\mathbf{r}, t)] \\
& +\nabla_{\mathbf{r}} \cdot \int \mathrm{d} \mathbf{p} \frac{\mathbf{p} \otimes \mathbf{p}}{m^{2}} f_{\text {neq }}^{(1)}(\mathbf{r}, \mathbf{p}, t),
\end{aligned}
$$

where the matrix given by the integral in the second term is trace-free. Using the identity

$$
\begin{aligned}
\partial_{t}(\rho(\mathbf{r}, t) \mathbf{v}(\mathbf{r}, t)) & +\nabla_{\mathbf{r}} \cdot[\rho(\mathbf{r}, t) \mathbf{v}(\mathbf{r}, t) \otimes \mathbf{v}(\mathbf{r}, t)] \\
= & \rho(\mathbf{r}, t) \partial_{t} \mathbf{v}(\mathbf{r}, t)+\left(\mathbf{v}(\mathbf{r}, t) \cdot \nabla_{\mathbf{r}}\right) \mathbf{v}(\mathbf{r}, t)
\end{aligned}
$$

(see e.g. $[42,(27)-(31)])$ in (8) produces the evolution equation

$$
\begin{aligned}
\rho\left(\mathbf{r}_{1}, t\right) \partial_{t} \mathbf{v}\left(\mathbf{r}_{1}, t\right) & +\rho\left(\mathbf{r}_{1}, t\right)\left(\mathbf{v}\left(\mathbf{r}_{1}, t\right) \cdot \nabla_{\mathbf{r}_{1}}\right) \mathbf{v}\left(\mathbf{r}_{1}, t\right) \\
+ & \boldsymbol{\nabla}_{\mathbf{r}_{1}} \cdot \int \mathrm{d} \mathbf{p}_{1} \frac{\mathbf{p}_{1} \otimes \mathbf{p}_{1}}{m^{2}} f_{\text {neq }}^{(1)}\left(\mathbf{r}_{1}, \mathbf{p}_{1}, t\right) \\
+ & \frac{1}{m} \rho\left(\mathbf{r}_{1}, t\right) \boldsymbol{\nabla}_{\mathbf{r}_{1}} \frac{\delta \mathcal{F}[\rho]}{\delta \rho}+\gamma \rho\left(\mathbf{r}_{1}, t\right) \mathbf{v}\left(\mathbf{r}_{1}, t\right) \\
+ & \frac{\gamma N}{m} \sum_{j=1}^{N} \int \mathrm{d} \mathbf{r}^{N-1} \mathrm{~d} \mathbf{p}^{N} \tilde{\boldsymbol{\Gamma}}_{1 j}\left(\mathbf{r}^{N}\right) \mathbf{p}_{j} f^{(N)}\left(\mathbf{r}^{N}, \mathbf{p}^{N}, t\right)=0 .
\end{aligned}
$$

There are a number of standard approaches to approximating the non-equilibrium term. We will focus on two, namely the Chapman-Enskog [59] and Grad [58] approximations. Both approximations make an expansion around the local-equilibrium distribution as $f^{(1)}=f_{\mathrm{le}}^{(1)}\left[1+\phi_{1}+\phi_{2}+\ldots\right]=f_{\mathrm{le}}^{(1)}+f_{\text {neq }}^{(1)}$. The difference lies in the choice of the $\phi_{i}$ 's. The aim is to construct a 'normal solution' for which all space and time dependence is implicitly described in terms of the hydrodynamic variables $\rho, \mathbf{v}$ and $T$ [56].

In the Chapman-Enskog method, it is assumed that the solution is close to local equilibrium, and $f^{(1)}$ is expanded in the gradients of $\rho, \mathbf{v}$ and $T$. This expansion is then truncated at a finite order. In Grad's model, the non-equilibrium term is expanded in moments (in $\mathbf{p}$ ) of the distribution function, i.e. in terms of projections onto Hermite polynomials. It is then standard to truncate the expansion such that only thirteen moments remain [58].

In this section, we take our lead from Grad's method for two reasons: (a) we wish to consider distributions which are not 'close' to local equilibrium and (b) the terms in (19) are naturally expressed as projections onto Hermite polynomials. In Section 5 we will demonstrate that the Chapman-Enskog method allows us to connect (19) with the Navier-Stokes equation. 
We begin with the generalized Hermite polynomials of one variable, $\left.H_{n}^{[\alpha]}=H_{n}^{[1]}(x / \sqrt{\alpha})\right) / \sqrt{n !}$, which are orthonormal with respect to the weight $(2 \pi \alpha)^{-1 / 2} \exp \left(-x^{2} /(2 \alpha)\right)$, giving a basis of $\mathbb{R}$. An orthonormal basis of $\mathbb{R}^{d}$ is then given, for $n \in \mathbb{N} \cup\{0\}$ and $\beta$ a vector of non-negative integers, by $P_{n, \beta}^{[\alpha]}\left(x_{1}, \ldots, x_{d}\right)=$ $H_{\beta_{1}}^{[\alpha]}\left(x_{1}\right) \ldots H_{\beta_{d}}^{[\alpha]}\left(x_{d}\right), \sum \beta_{i}=n$ (see e.g. [60]; this follows from $\mathbb{R}^{d}$ being a product space). For reference, in three dimensions and with $\mathbf{e}_{j}$ the standard unit vectors for $\mathbb{R}^{3}$, the first few $P_{n, \beta}^{[\alpha]}$ are given by $P_{0,(0,0,0)}^{[\alpha]}=1, P_{1, \mathbf{e}_{j}}^{[\alpha]}=x_{j} / \sqrt{\alpha}$, and $P_{1,\left(\mathbf{e}_{j}+\mathbf{e}_{k}\right)}^{\left[{ }^{2}\right.}=x_{j} x_{k} / \alpha-\delta_{j k}$. We now set $\alpha=m k_{B} T$ and write $f^{(1)}(\mathbf{r}, \mathbf{p}, t)=f_{\text {le }}^{(1)}(\mathbf{r}, \mathbf{p}, t)(1+\Phi(\mathbf{r}, \mathbf{p}, t))$ with $f_{\text {le }}$ as in (10) and $\Phi(\mathbf{r}, \mathbf{p}, t)=\sum_{n, \beta} B_{n, \beta}(\mathbf{r}, t) P_{n, \beta}^{[\alpha]}(\mathbf{p}-m \mathbf{v}(\mathbf{r}, t))$. The requirements on $f_{\text {neq }}^{(1)}$ imposed by (11)-(16) then reduce to $B_{0,\{0,0,0\}}=B_{1, \mathbf{e}_{j}}=0, j=1,2,3$ and $\sum_{j} B_{2,2 \mathbf{e}_{j}}=0$. Furthermore, by the orthonormality of the Hermite polynomials, $\left[\int \mathrm{d} \mathbf{p}(\mathbf{p} \otimes \mathbf{p}) f_{\text {neq }}^{(1)}(\mathbf{r}, \mathbf{p}, t)\right]_{i, j}=m k_{B} T \rho(\mathbf{r}, t) B_{2, \mathbf{e}_{i}+\mathbf{e}_{j}}$, irrespective of if the expansion in Hermite polynomials is truncated or not.

In particular, the kinetic pressure term need not be small out of local equilibrium as the $B_{2, \mathbf{e}_{i}+\mathbf{e}_{j}}$ are completely independent of $\rho, \mathbf{v}$ and $T$. To understand the evolution of this term, it would be necessary to consider the energy equations (found by multiplying (2) by $p_{i} p_{j}$ and integrating). To do so would require knowledge of the terms in $f^{(1)}$ proportional to $P_{3, \beta}$, again leading to an infinite hierarchy of equations, which must be truncated. In Section 6 we show that the kinetic pressure term may be neglected in the limit of large friction, i.e. $\gamma \gg 1$; see [44] for a rigorous study of this regime. Assuming for the moment that we may approximate or neglect the term containing $f_{\text {neq }}^{(1)}$, in order to obtain a DDFT, it remains to treat the friction tensor term in (19).

\subsection{Two-body friction and the Enskog closure}

For this section we restrict our analysis to the case where $\tilde{\boldsymbol{\Gamma}}_{i j}$ are given by linear combinations of two-body interactions. This is analogous to the two-body restriction on the diffusion tensor in [25]. However, it is not in general the case that if $\mathbf{D}$ contains only two-body interactions then so does $\boldsymbol{\Gamma}$. This can be seen from the definition $\mathbf{D}=k_{B} T / m \boldsymbol{\Gamma}^{-1}$, noting that each element of the inverse will include the inverse of the determinant, which contains all entries of $\mathbf{D}$, and hence will in general be $N$-body. This restriction to two-body terms can be lifted if one can obtain accurate approximate functional relationships between $f^{(n)}$ and $f^{(1)}, n=3, \ldots N$, as we now discuss for $f^{(2)}$ (see (20)). We note that, for unbounded, Stokes-flow baths, general two-body expansions are available for both the diffusion and friction tensors, which are valid for all separations [61]. It is worth noting that the two-body formulation of the friction tensor is generally more accurate than that of the diffusion tensor, which can lead to unphysical effects [62].

After the restriction to two-body HI, (19) contains only $f^{(2)}\left(\mathbf{r}_{1}, \mathbf{p}_{1}, \mathbf{r}_{2}, \mathbf{p}_{2}, t\right)$. We make the Enskog approximation

$$
f^{(2)}\left(\mathbf{r}_{1}, \mathbf{p}_{1}, \mathbf{r}_{2}, \mathbf{p}_{2}, t\right)=f^{(1)}\left(\mathbf{r}_{1}, \mathbf{p}_{1}, t\right) f^{(1)}\left(\mathbf{r}_{2}, \mathbf{p}_{2}, t\right) g\left(\mathbf{r}_{1}, \mathbf{r}_{2},[\rho]\right),
$$


where in particular we have defined there to be no explicit momentum correlation $[63,64,65]$. Despite this, as we will see, the DDFT (23) still retains momentum correlations through the HI tensor. Going beyond this approximation introduces additional coupling between the momentum moment equations, analogous to the term in (19) containing $f_{\text {neq }}$, which must then also be approximated. The functional dependence of $f^{(2)}$ on $\rho$ can be rigorously justified from the fact [13] that the full time-dependent $N$ body distribution is a functional of $\rho$ [13], and hence so are all the reduced distributions. However, as with the excess over ideal gas term, the exact form of this functional is unknown.

We now assume that we know $g$ exactly, or have a good approximation for it (see the end of this section for further details) and write

$$
\tilde{\boldsymbol{\Gamma}}_{i j}\left(\mathbf{r}^{N}\right)=\delta_{i j} \sum_{\ell \neq i} \mathbf{Z}_{1}\left(\mathbf{r}_{i}, \mathbf{r}_{\ell}\right)+\left(1-\delta_{i j}\right) \mathbf{Z}_{2}\left(\mathbf{r}_{i}, \mathbf{r}_{j}\right),
$$

which is the most general form of $\tilde{\boldsymbol{\Gamma}}_{i j}$ under the assumptions that $\boldsymbol{\Gamma}$ is invariant under interchange of particle labelling, that there are only two-body interactions, and that the force on particle $i$ caused by the momentum of particle $j$ is independent of the positions of the remaining particles. Inserting (21) into (19) and performing the remaining $\mathbf{p}$ integrals in the HI term gives

$$
\begin{aligned}
& \rho\left(\mathbf{r}_{1}, t\right) \partial_{t} \mathbf{v}\left(\mathbf{r}_{1}, t\right)+\rho\left(\mathbf{r}_{1}, t\right)\left(\mathbf{v}\left(\mathbf{r}_{1}, t\right) \cdot \nabla_{\mathbf{r}_{1}}\right) \mathbf{v}\left(\mathbf{r}_{1}, t\right) \\
& +\boldsymbol{\nabla}_{\mathbf{r}_{1}} \cdot \int \mathrm{d} \mathbf{p}_{1} \frac{\mathbf{p}_{1} \otimes \mathbf{p}_{1}}{m^{2}} f_{\text {neq }}^{(1)}\left(\mathbf{r}_{1}, \mathbf{p}_{1}, t\right) \\
& +\frac{1}{m} \rho\left(\mathbf{r}_{1}, t\right) \boldsymbol{\nabla}_{\mathbf{r}_{1}} \frac{\delta \mathcal{F}[\rho]}{\delta \rho}+\gamma \rho\left(\mathbf{r}_{1}, t\right) \mathbf{v}\left(\mathbf{r}_{1}, t\right) \\
& +\gamma \rho\left(\mathbf{r}_{1}, t\right) \int \mathrm{d} \mathbf{r}_{2}\left[\mathbf{Z}_{1}\left(\mathbf{r}_{1}, \mathbf{r}_{2}\right) \mathbf{v}\left(\mathbf{r}_{1}, t\right)+\mathbf{Z}_{2}\left(\mathbf{r}_{1}, \mathbf{r}_{2}\right) \mathbf{v}\left(\mathbf{r}_{2}, t\right)\right] \\
& \times \rho\left(\mathbf{r}_{2}, t\right) g\left(\mathbf{r}_{1}, \mathbf{r}_{2},[\rho]\right)=0 .
\end{aligned}
$$

Finally, neglecting the $f_{\text {neq }}^{(1)}$ term as described in the previous section and dividing throughout by $\rho\left(\mathbf{r}_{1}, t\right)$, we obtain

$$
\begin{aligned}
\partial_{t} \mathbf{v}\left(\mathbf{r}_{1}, t\right)+\left(\mathbf{v}\left(\mathbf{r}_{1}, t\right) \cdot \nabla_{\mathbf{r}_{1}}\right) \mathbf{v}(\mathbf{r}, t)+\frac{1}{m} \nabla_{\mathbf{r}_{1}} \frac{\delta \mathcal{F}[\rho]}{\delta \rho}+\gamma \mathbf{v}\left(\mathbf{r}_{1}, t\right) \\
+\gamma \int \mathrm{d} \mathbf{r}_{2}\left[\mathbf{Z}_{1}\left(\mathbf{r}_{1}, \mathbf{r}_{2}\right) \mathbf{v}\left(\mathbf{r}_{1}, t\right)+\mathbf{Z}_{2}\left(\mathbf{r}_{1}, \mathbf{r}_{2}\right) \mathbf{v}\left(\mathbf{r}_{2}, t\right)\right] \\
\times \rho\left(\mathbf{r}_{2}, t\right) g\left(\mathbf{r}_{1}, \mathbf{r}_{2},[\rho]\right)=0 .
\end{aligned}
$$

This, combined with the continuity equation

$$
\partial_{t} \rho\left(\mathbf{r}_{1}, t\right)+\nabla_{\mathbf{r}_{1}} \cdot\left(\rho\left(\mathbf{r}_{1}, t\right) \mathbf{v}\left(\mathbf{r}_{1}, t\right)\right)=0,
$$

gives a DDFT for $\rho$, which is our main result. In particular, we recover the DDFT of [42] by setting $\mathbf{Z}_{1}=\mathbf{Z}_{2} \equiv 0$.

We now discuss the physical interpretation of the terms depending on the $\mathbf{Z}_{i}$. The first term in the integral in (23) may be combined with the $\gamma \mathbf{v}$ to give a densitydependent effective friction coefficient. If $\mathbf{Z}_{2} \equiv 0$ then this is the only additional term, 
and the equation is non-local in $\rho$ and local in $\mathbf{v}$, which is expected, as it is the offdiagonal terms in $\boldsymbol{\Gamma}$ that couple the momenta of different colloid particles. A non-zero $\mathbf{Z}_{2}$ introduces non-localities in $\mathbf{v}$ representing, at a mean-field level, the coupling of velocities of different particles, which is retained despite making the Enskog approximation (20). As shown in [44], since $\boldsymbol{\Gamma}$ is positive-definite,

$$
\begin{aligned}
\mathbf{v}\left(\mathbf{r}_{1}, t\right) \cdot \gamma\left[\mathbf{1}+\int \mathrm{d} \mathbf{r}_{2}\right. & {\left[\mathbf{Z}_{1}\left(\mathbf{r}_{1}, \mathbf{r}_{2}\right) \mathbf{v}\left(\mathbf{r}_{1}, t\right)+\mathbf{Z}_{2}\left(\mathbf{r}_{1}, \mathbf{r}_{2}\right) \mathbf{v}\left(\mathbf{r}_{2}, t\right)\right] } \\
& \left.\times \rho\left(\mathbf{r}_{2}, t\right) g\left(\mathbf{r}_{1}, \mathbf{r}_{2},[\rho]\right)\right]>0,
\end{aligned}
$$

and the overall effect is a friction-like retardation of velocity.

It is well-known that hydrodynamic interactions (here described by the $\mathbf{Z}_{i}$ 's) are long range, typically decaying as the inverse of the particle separation. This calls into question the convergence of the integrals in (23), the integrand of which, for arbitrary $\mathbf{v}$, $\rho$ and $g$, does not have sufficiently rapid decay. However, we assume (as is done in the derivation of the Rotne-Prager tensor) that the velocity and density have sufficient decay as $|\mathbf{r}| \rightarrow \infty$ for the integrals to be well-defined. This is certainly physically reasonable for systems with a finite number of particles. In particular, for confined systems the density and velocity are both zero outside some finite volume.

It remains to determine the correlation function $g\left(\mathbf{r}_{1}, \mathbf{r}_{2},[\rho]\right)$, i.e. to explicitly close (23). Under the adiabatic approximation, it is determined by the Ornstein-Zernike equation [14] $g\left(\mathbf{r}_{1}, \mathbf{r}_{2}\right)=1+c^{(2)}\left(\mathbf{r}_{1}, \mathbf{r}_{2}\right)+\int \mathrm{d} \mathbf{r}_{3}\left[g\left(\mathbf{r}_{1}, \mathbf{r}_{3}\right)-1\right] \rho\left(\mathbf{r}_{3}\right) c^{(2)}\left(\mathbf{r}_{2}, \mathbf{r}_{3}\right)$, where

$c^{(2)}\left(\mathbf{r}_{1}, \mathbf{r}_{2}\right)=\frac{\delta^{2} \mathcal{F}_{\text {exc }}[\rho]}{\delta \rho\left(\mathbf{r}_{1}, t\right) \delta \rho\left(\mathbf{r}_{2}, t\right)}$. We recall that, in general, $\mathcal{F}_{\text {exc }}[\rho]$ is not known explicitly. The exception is for hard rods in one dimension, in which case $g$ may be computed (numerically) with no further assumptions. Whilst in principle one can obtain $g$ from these two equations, in practice it is useful to assume that $g\left(\mathbf{r}_{1}, \mathbf{r}_{2},[\rho]\right)$ may be wellapproximated by some known $\tilde{g}\left(\left|\mathbf{r}_{1}-\mathbf{r}_{2}\right|, \eta\right)$, where $\eta$ is a scalar parameter such as the average packing fraction. This additional approximation neglects anisotropies of the system, both in the averaging procedure and in the reduction to dependence on particle separation. The advantage for a hard-sphere fluid is that an analytic expression for $\tilde{g}$ has been derived via the Percus-Yevick equation. We also note that the density-dependence of $g$ introduces an implicit time-dependence. However, as we will see in the next section, it is often sufficient to choose a simple approximation to $g$. We further note that it is possible to derive an equation of motion for $g$ by integrating 2 over all but two positions. However, this doubles the dimension of the resulting problem, and requires additional closure schemes.

Before discussing approximations to $f_{\text {neq }}$ in (22), we verify our DDFT via numerical experiments under the assumption that $f_{\text {neq }}=0$.

\section{Verification and Numerical Experiments}

The purpose of this section is twofold. Firstly we demonstrate that, at least for the model systems investigated here, the unconstrained approximations made in deriving (23) 
produce an accurate DDFT. Secondly, the numerical implementation of such DDFTs is non-trivial, but must be performed for the DDFT to be more than a theoretical tool.

We consider four pairs of simulations, which contrast various DDFT with the underlying stochastic dynamics, and the effects of inertia and HI. The first pair of simulations accounts for both inertia and HI and consists of our DDFT (23) and (24) and the Euler-Maruyama [66] solution of the full Langevin equations (1). The second pair of simulations includes inertia but neglects HI by solving the same two sets of equations but with the HI tensor $\tilde{\boldsymbol{\Gamma}} \equiv 0$. This is the special case previously derived by Archer [42]. The third pair neglects inertia but retains HI in the form of a two-body diffusion tensor, with the DDFT derived by Rex and Löwen [25] compared to the ErmakMcCammon [45] solution of the corresponding configuration-space stochastic equations. The final pair neglects both inertia and HI by choosing the diffusion tensor to be a scalar multiple of the identity in the DDFT and stochastic equations. This corresponds to the original DDFT of Marconi and Tarazona [21].

For the whole of this section we non-dimensionalize the equations with the units of length, mass and energy being $\sigma, m$ and $k_{B} T$, respectively. We also fix the dimensionless friction coefficient $\gamma=2$ but note that we have tested the formalism for a wide range of $\gamma$ and choice of potentials. The specific choice of $\gamma$ is relatively unimportant. For increasingly larger values of $\gamma$, the dynamics approach those of the more standard overdamped DDFTs, whilst for increasingly smaller values the effects of inertia are more obvious, but the approximation of neglecting $f_{\text {neq }}$ can lead to numerical instabilities for very small values. These numerical issues suggest that the DDFT presented here is not applicable for molecular fluids which have $\gamma=0$.

We choose the colloidal particles to be hard spheres, with $V_{2}\left(\left|\mathbf{r}-\mathbf{r}^{\prime}\right|\right)=\infty$ for $\left|\mathbf{r}-\mathbf{r}^{\prime}\right|<1$ and zero otherwise. For the stochastic calculations, where the potential must be differentiable, we use a slightly softened potential $V_{2}\left(\left|\mathbf{r}-\mathbf{r}^{\prime}\right|\right)=$ $\left|\mathbf{r}-\mathbf{r}^{\prime}\right|^{-48}-\left|\mathbf{r}-\mathbf{r}^{\prime}\right|^{-24}+1 / 4$ if $\left|\mathbf{r}-\mathbf{r}^{\prime}\right|<2^{1 / 24}$ and zero otherwise. For the DDFT calculations, we choose $\mathcal{F}$ to be either the exact $1 \mathrm{D}$ hard rod functional first derived by Percus [48], or the approximate three-dimensional FMT functional of Rosenfeld [49] as appropriate. Whilst, as discussed above, it is possible to obtain the equilibrium correlation functional $g$ from the Ornstein-Zernike equation [14], we choose $g$ to be the simplest possible hard sphere approximation, $g\left(\left|\mathbf{r}-\mathbf{r}^{\prime}\right|\right)=0$ for $\left|\mathbf{r}-\mathbf{r}^{\prime}\right|<1$ and unity otherwise. This significantly simplifies the DDFT calculation and, as we will see, has little effect on the quality of the results. This approximation is more accurate for diffuse systems, and is likely to break down when the particles are tightly packed.

For the diffusion tensor in the configuration space calculations, we choose the same Rotne-Prager two-body approximation as used in [25], i.e. $\mathbf{D}=\left(\mathbf{D}_{i j}\right)$ with

$$
\begin{aligned}
\mathbf{D}_{i j}\left(\mathbf{r}^{N}\right)=\gamma^{-1}\left[\delta_{i j} \mathbf{1}\right. & +\delta_{i j} \sum_{\ell \neq i}^{N} \mathbf{D}_{11}\left(\mathbf{r}_{i}-\mathbf{r}_{\ell}\right) \\
& \left.+\left(1-\delta_{i j}\right) \mathbf{D}_{12}\left(\mathbf{r}_{i}-\mathbf{r}_{j}\right)\right] .
\end{aligned}
$$

We also introduce a hydrodynamic diameter $\sigma_{\mathrm{H}}<\sigma$ which allows us to neglect 
lubrication forces present when particles are close to each other. In addition, this parameter increases the accuracy of the two-body approximation for the friction tensor that we shall discuss shortly. Such an effective diameter is appropriate for many commonly-studied colloidal particles which consist of a hard core with a layer of polymer; see e.g. [67]. We choose $\sigma_{\mathrm{H}}=0.5$, but good agreement was found between the DDFTs and stochastic calculations containing $\mathrm{HI}$ for $\sigma_{\mathrm{H}} \lesssim 0.75$. The DDFTs without $\mathrm{HI}$ are independent of this choice. Our choice for the diffusion tensor is

$$
\mathbf{D}_{12}(\mathbf{r})=\frac{3}{8}\left(\frac{\sigma_{\mathrm{H}}}{|\mathbf{r}|}\right)\left[\mathbf{1}+\frac{\mathbf{r} \otimes \mathbf{r}}{|\mathbf{r}|^{2}}\right]+\frac{1}{16}\left(\frac{\sigma_{\mathrm{H}}}{|\mathbf{r}|}\right)^{3}\left[\mathbf{1}-3 \frac{\mathbf{r} \otimes \mathbf{r}}{|\mathbf{r}|^{2}}\right]
$$

and $\mathbf{D}_{11}=\mathcal{O}\left(1 / r^{4}\right)$ and so, for diffuse systems, is negligible.

The full tensor $\mathbf{D}$ is positive-definite (which is required for the stochastic equations) and two-body. In contrast, there is no such standard formulation for the friction tensor. We therefore choose $\boldsymbol{\Gamma}=\mathbf{D}^{-1}$, which is also positive-definite. For the twobody approximation required by the DDFT, we use the 11-term expansion given by Jeffrey and Onishi [61], under the assumption that $\sigma_{\mathrm{H}} /\left|\mathbf{r}_{i}-\mathbf{r}_{j}\right|$ is small. Although not strictly equivalent, we will see that these two approximations are in good quantitative and excellent qualitative agreement, but exact agreement should not be expected.

As mentioned previously, the Langevin equations are solved using the EulerMaruyama scheme [66] for phase space calculations and the Ermak-McCammon scheme [45] for the configuration space calculations. We average over 5000 simulations where the step size is chosen such that halving it produces no appreciable change in the results. The DDFTs are solved using spectral methods [68], suitably extended to integrodifferential equations along with a fifth-order implicit Runge-Kutta method with step size control[69].

We first consider a 1D system consisting of 8 hard rod particles initially at equilibrium in a confining quadratic external potential $V_{1}(z)=0.01 z^{2}$, which is not strong enough to force layering effects. As such, the difference between the grand canonical DDFT formalisms and the canonical stochastic equations is minimized, cf. Figure 1 of [21] where the difference for strongly-confining potentials is large. At time $t=0$ the external potential is instantaneously switched to one of the form $V_{1}(z, t)=0.01 z^{2}+20 \exp \left(-\left(z-z_{0}(t)\right)^{2} / 40\right)$ where $z_{0}(t)=12 \sin (t \pi / 8)$, which consists of the original quadratic potential and a Gaussian bump, the mean position of which oscillates in time. The precise external potentials chosen in this section are unimportant; we have found very good agreement between the DDFTs and stochastic simulations for a wide range of potentials. They are chosen to (exponentially) confine the density, but without hard walls, which drastically increase the complexity of the HI and which will be the focus of future work. These can be thought of as simple models for physical systems such as particles confined by an optical trap, as in [25]. Dynamics are then induced by a time-dependence of the external field.

In Figure 1 we show the position $\rho(z)$ and velocity $v(z)$ distributions for various times. The agreement between the DDFT and stochastic simulations is very good in all four cases. In addition, there is a qualitative difference between the four 
pairs of simulations, demonstrating the importance of both inertial effects and HI. Quantitatively, we note that in this case HI appear to reduce the overall friction of the system. Whilst the velocity profiles with and without HI are similar, those when HI are included have higher absolute velocities. In Figure 2 we plot the change in the number of particles between $z=-8$ and $z=8$ (i.e. $\int_{-8}^{8} \mathrm{~d} z \rho(z, t)$ ) over time. Recall that the system is unconfined, but the density decays exponentially quickly to zero. Once again, the agreement between DDFT and stochastic calculations is very good and one sees the large quantitative differences produced by including $\mathrm{HI}$.
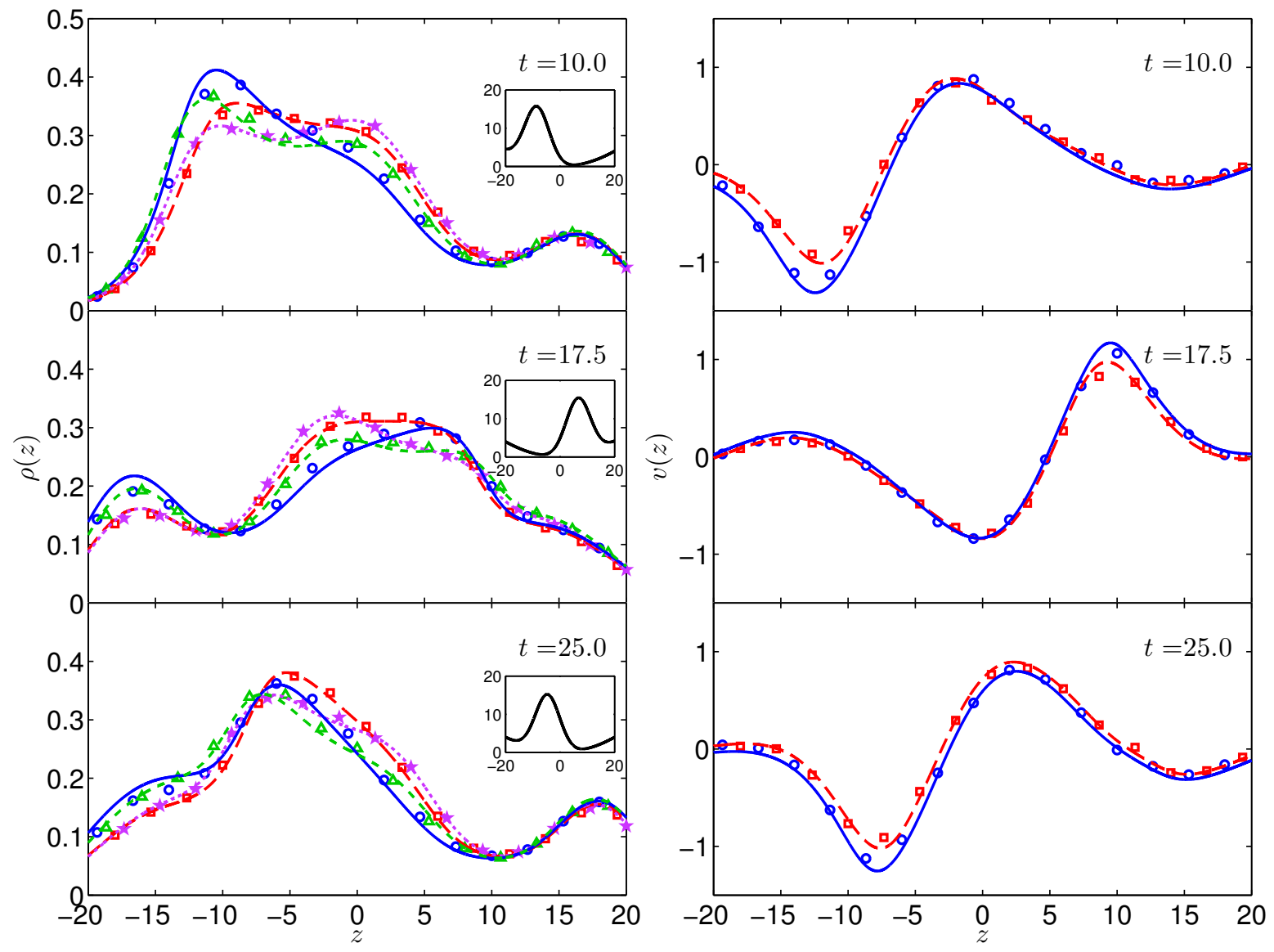

Figure 1. Comparison of four different DDFTs (lines) and the corresponding underlying stochastic simulations (symbols) for 8 hard rods in an oscillating potential (see text). Full phase space with (blue, solid, circles) and without (red, long dashes, squares) HI and the overdamped approximation with (green, short dashes, triangles) and without (purple, dotted, stars) HI. Left column shows particle distribution as a function of position at three representative times. Right column shows the corresponding velocity distributions for the phase space calculations. Insets show the external potential as a function of $z$ at each time. Note the excellent agreement between the DDFT and stochastic calculations, as well as the qualitative differences introduced by the inclusion of inertia and/or HI.

We next study a 3D radially symmetric system where, for $r=|\mathbf{r}|, V_{1}(r, t)=$ $0.1 r^{2}(1-h(r, t))-\beta \exp \left[\left(r-r_{0}(t)\right)^{2} / \alpha^{2}\right]$ where $h(r, t)=1 / 2\left[\operatorname{erf}\left[\left(r+r_{0}(t) / \alpha\right]-\operatorname{erf}[(r-\right.\right.$ 


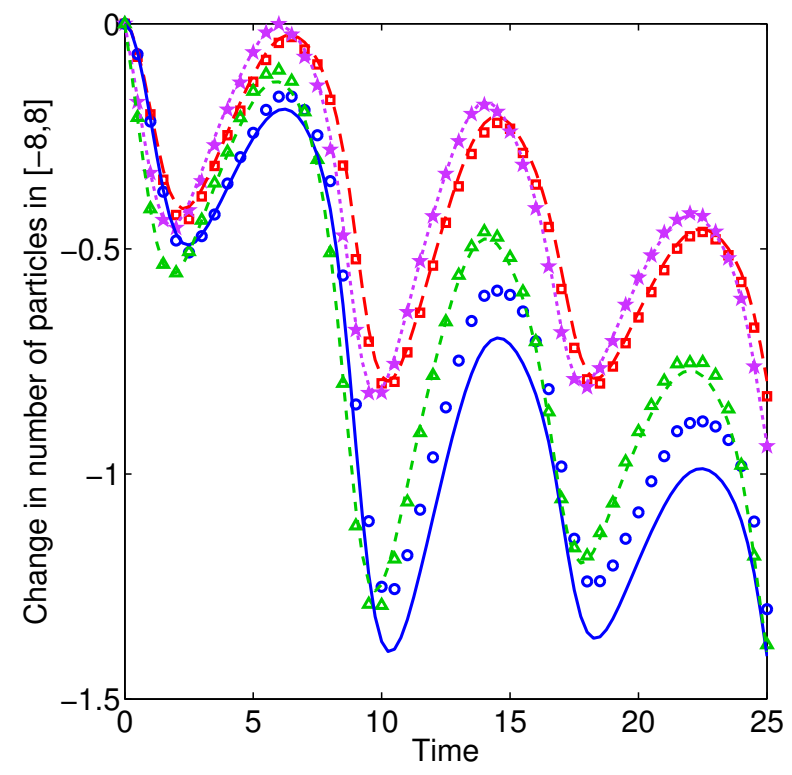

Figure 2. Change in the number of particles in $[-8,8]$ from four DDFTs (lines) and corresponding stochastic simulations (symbols) for 8 hard rods in an oscillating potential (see text). Lines and symbols as in Figure 1.

$\left.\left.r_{0}(t) / \alpha\right]\right]$ is a smooth cutoff function. As such, the density depends only on the radial coordinate and the DDFT may be reduced to a $1 \mathrm{D}$ calculation. We consider first 50 particles with $\alpha=4, \beta=10$ and $r_{0}(t)=3+\sin (t \pi)$, where the particles are initially at equilibrium under potential $V_{1}(r, 0)$. Figure 3a shows the evolution of the mean radial position and velocity for the four pairs of simulations. In contrast to the apparent reduction in friction when including $\mathrm{HI}$ in the $1 \mathrm{D}$ system above, here the $\mathrm{HI}$ introduce damping effects. As can be seen, including inertia results in a slower initial motion of the centre of mass and also affects the period of the dynamics.

To further illustrate these effects, in Figure $3 \mathrm{~b}$ we consider the same system but with $r_{0}(t)=4$ for $t>0$, i.e. evolution towards equilibrium. Again we observe a qualitatively different behaviour of the dynamics, as well as the retardation effects of HI. Once again, the agreement between the DDFT and stochastic simulations is very good.

Having established the very good agreement between each of the DDFTs and appropriate underlying stochastic equations, and demonstrated the importance of including both inertia and HI, we now demonstrate the computational convenience of applying DDFT to a large number of particles. We consider the same dynamics for 1000 particles with $\alpha=9, \beta=10$ and $r_{0}(t)=14+\sin (t \pi)$, which are chosen so that the maximum density is approximately the same as that for the 50 particle system. In particular, it is important to note that the computational cost for solving the system with 1000 particles is identical to that with 50 particles, whereas solving the stochastic equations would be $\mathcal{O}\left(10^{3}\right)$ times more expensive. Figure 4 demonstrates that the behaviour of the larger system is similar to that of the 50 particle system. One quantitative difference to be noted is the greater relative impact of HI. From this 

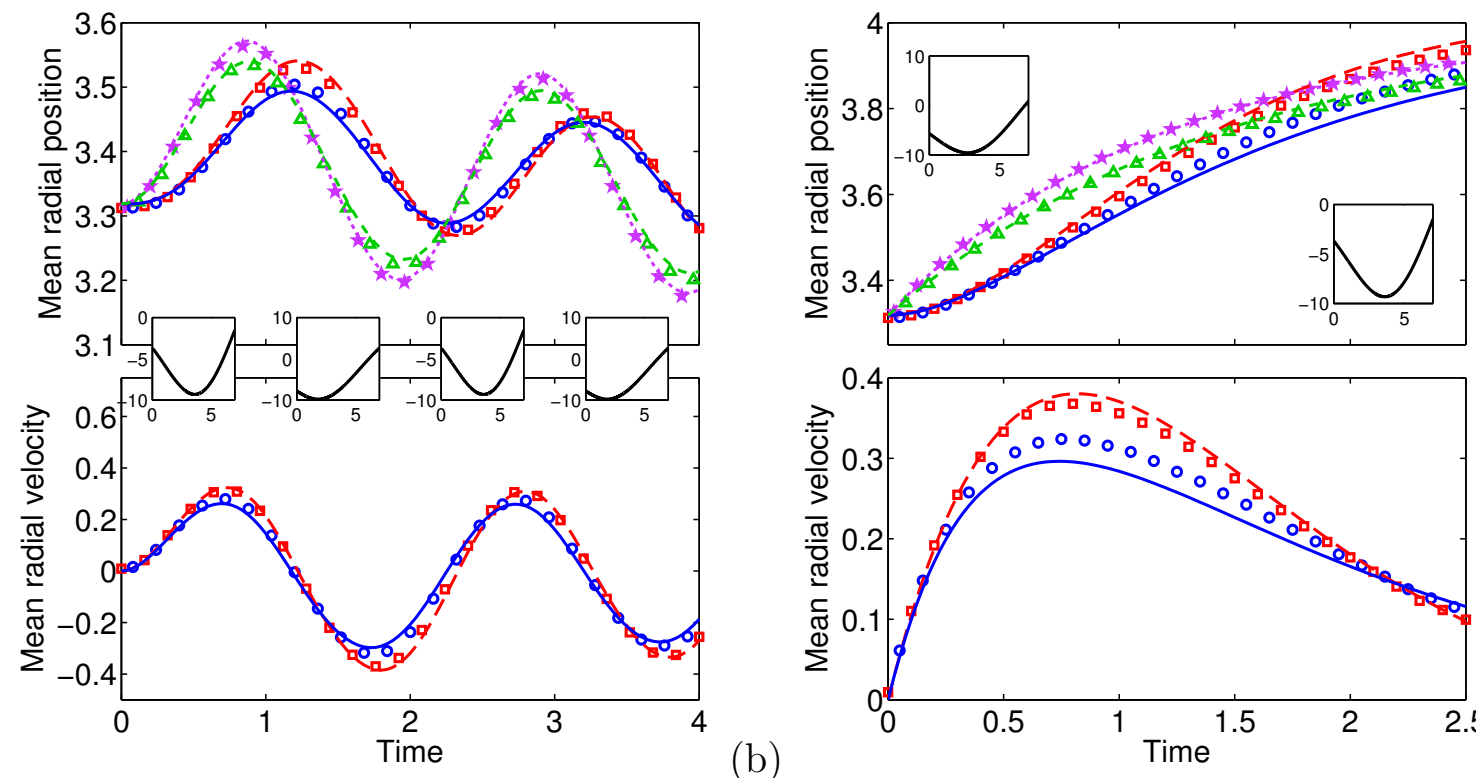

(a)

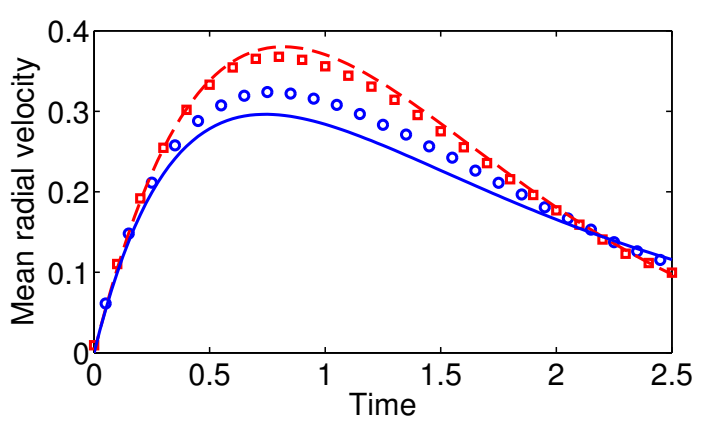

Figure 3. Comparison of four DDFTs (lines) and corresponding stochastic simulations (symbols) for 50 hard spheres in two different radially-symmetric potentials (see text). Top plots show the mean radial position of the 50 particles against time, bottom plots the corresponding mean radial velocity. Lines and symbols as in Figure 1. Insets in (a) show the external potential as a function of the radial position at times $0.5,1.5,2.5$ and 3.5. Those in (b) show the external potential for time 0 (top left) and all positive times (bottom right). Note the excellent agreement between the DDFT and stochastic calculations, as well as the qualitative differences introduced by the inclusion of inertia and the quantitative damping effects of HI.

we would expect HI to become increasingly significant in systems with a macroscopic number of particles. However, it is important to note that the HI models used here neglect effects such as screening and finite-propagation time, which may become important for large macroscopic systems. For further demonstrations of the effects of inertia and HI in 3D hard sphere systems, see [43].

\section{Connection to the Navier-Stokes equation}

For this section, we restrict our attention to the case where the potential includes at most two-body terms and assume $V_{2}$ depends only on the separation of the two particles, $V_{2}\left(\mathbf{r}_{1}, \mathbf{r}_{2}\right)=V_{2}\left(\left|\mathbf{r}_{1}-\mathbf{r}_{2}\right|\right)$. We return to (22) and suppose, in the spirit of the ChapmanEnskog expansion, that the system is close to local equilibrium so that $f_{\text {neq }}^{(1)}$ and $\rho^{(2)}$ can be expanded in powers of $\boldsymbol{\nabla}_{\mathbf{r}} \mathbf{v}$. Our analysis follows [56, Section 7.4.1]. Enforcing (11)(16) and noting that the system is isotropic, we have the truncated Taylor expansion

$$
\begin{aligned}
f^{(1)}(\mathbf{r}, \mathbf{p}, t)= & f_{\mathrm{le}}^{(1)}(\mathbf{r}, \mathbf{p}, t) \\
& +A_{1}(|\mathbf{p}-m \mathbf{v}|)\left[\frac{(\mathbf{p}-m \mathbf{v}) \cdot \boldsymbol{\Lambda} \cdot(\mathbf{p}-m \mathbf{v})}{\left.|\mathbf{p}-m \mathbf{v}|^{2}\right)}-\frac{1}{3} \boldsymbol{\nabla}_{\mathbf{r}} \cdot \mathbf{v}\right]
\end{aligned}
$$




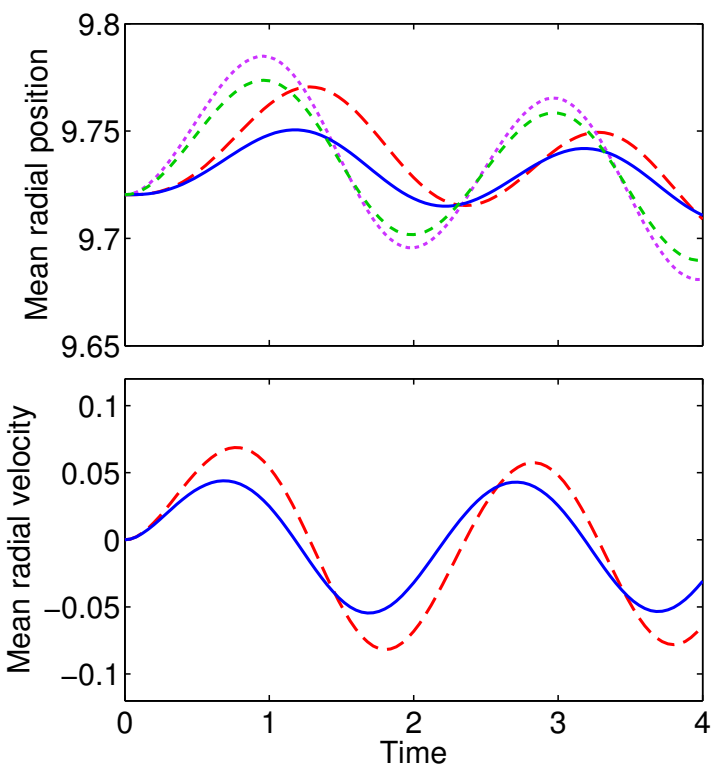

Figure 4. Mean radial position and velocity for 1000 hard sphere particles, as computed for four different DDFTs. Lines as in Figure 1. Insets show the external potential as a function of the radial position at times $0.5,1.5,2.5$ and 3.5.

where $\boldsymbol{\Lambda}_{\alpha, \beta}=\frac{1}{2}\left(\frac{\partial v_{\alpha}}{\partial r_{\beta}}+\frac{\partial v_{\beta}}{\partial r_{\alpha}}\right)$ and $f_{\mathrm{le}}^{(1)}$ is given by (10). Using (11)-(15) gives $\mathbf{A}(\mathbf{r}, t)=\mathbf{A}_{\mathrm{le}}(\mathbf{r}, t)$

$$
+\frac{1}{m^{2}} \boldsymbol{\nabla}_{\mathbf{r}} \cdot \int \mathrm{d} \mathbf{p}(\mathbf{p} \otimes \mathbf{p}) A_{1}(|\mathbf{p}|)\left[\frac{\mathbf{p} \cdot \boldsymbol{\Lambda} \cdot \mathbf{p}}{|\mathbf{p}|^{2}}-\frac{1}{3} \boldsymbol{\nabla}_{\mathbf{r}} \cdot \mathbf{v}(\mathbf{r}, t)\right],
$$

where $\mathbf{A}_{\text {le }}$ is given by (17) with $f_{\text {neq }}=0$.

We now use the identity [56]

$$
\begin{aligned}
\int \mathbf{p} \cdot(F(|\mathbf{p}|) & \left.-\frac{1}{3} \operatorname{Tr}(|\mathbf{p}|) \mathbf{1}\right) \cdot \mathbf{p} \frac{\mathbf{p} \otimes \mathbf{p}}{|\mathbf{p}|^{2}} \mathrm{~d} \mathbf{p} \\
& =\frac{2}{15} \int\left(F(|\mathbf{p}|)-\frac{1}{3} \operatorname{Tr}(|\mathbf{p}|) \mathbf{1}\right)|\mathbf{p}|^{2} \mathrm{~d} \mathbf{p},
\end{aligned}
$$

which is easy to prove using spherical polar coordinates, to rewrite (29) as

$$
\begin{aligned}
\mathbf{A}(\mathbf{r}, t)=\boldsymbol{\nabla}_{\mathbf{r}} \cdot & (\rho(\mathbf{r}, t) \mathbf{v}(\mathbf{r}, t) \otimes \mathbf{v}(\mathbf{r}, t)) \\
& -\frac{2}{m} \eta^{(K)} \boldsymbol{\nabla}_{\mathbf{r}} \cdot\left[\boldsymbol{\Lambda}-\frac{1}{3} \boldsymbol{\nabla}_{\mathbf{r}} \cdot \mathbf{v}\right],
\end{aligned}
$$

where $\eta^{(K)}:=-\frac{1}{15 m} \int \mathrm{d} \mathbf{p} A_{1}(|\mathbf{p}|)|\mathbf{p}|^{2}$. This corresponds to the kinetic part of the viscosity in the Navier-Stokes equation. We now derive the potential part by considering corrections to the two-body density.

Consider the term in (6) that depends on $V_{2}$, which me may write as $\frac{1}{m} \int \mathrm{d} \mathbf{r}_{2} \boldsymbol{\nabla}_{\mathbf{r}_{1}} V_{2}\left(\mathbf{r}_{1}, \mathbf{r}_{2}\right) \rho^{(2)}\left(\mathbf{r}_{1}, \mathbf{r}_{2}, t\right)=: \boldsymbol{\nabla}_{\mathbf{r}} \cdot \mathbf{P}^{(V)}$, where, following [56, Section 7.3.2] or [70], we find, for $\mathbf{r}_{12}^{\prime}=\mathbf{r}_{1}^{\prime}-\mathbf{r}_{2}^{\prime}, r_{12}^{\prime}=\left|\mathbf{r}_{12}^{\prime}\right|$,

$$
\mathbf{P}^{(V)}=-\frac{1}{2 m} \int \mathrm{d} \mathbf{r}_{12}^{\prime} n_{2}\left(\mathbf{r}_{1}, \mathbf{r}_{12}^{\prime}, t\right) \frac{\mathbf{r}_{12}^{\prime} \otimes \mathbf{r}_{12}^{\prime}}{r_{12}^{\prime}} \frac{\mathrm{d}}{\mathrm{d} r_{12}^{\prime}} V_{2}\left(r_{12}^{\prime}\right),
$$


with $n_{2}\left(\mathbf{r}_{1}, \mathbf{r}_{12}^{\prime}, t\right)=\int_{0}^{1} \mathrm{~d} \lambda \rho_{2}\left(\mathbf{r}_{1}+(1-\lambda) \mathbf{r}_{12}^{\prime}, \mathbf{r}_{1}-\lambda \mathbf{r}_{12}^{\prime}\right)$. We now use the expansion [56, (7.77)] corrected to

$$
\begin{aligned}
n_{2}\left(\mathbf{r}_{1}, \mathbf{r}_{12}^{\prime}, t\right)= & n_{2}^{(0)}\left(r_{12}^{\prime}, t\right) \\
& +B_{1}\left(r_{12}^{\prime}, t\right)\left(\frac{\mathbf{r}_{12}^{\prime} \cdot \boldsymbol{\Lambda} \cdot \mathbf{r}_{12}^{\prime}}{r_{12}^{\prime 2}}-\frac{1}{3} \nabla_{\mathbf{r}_{1}} \cdot \mathbf{v}\right) \\
& +B_{2}\left(r_{12}^{\prime}\right) \boldsymbol{\nabla}_{\mathbf{r}_{1}} \cdot \mathbf{v}
\end{aligned}
$$

where there are no restrictions on $B_{1}$ or $B_{2}$. These coefficients $B_{1}$ and $B_{2}$ are, respectively, the coefficients of the trace-free and trace contributions to the firstorder Taylor expansion in $\boldsymbol{\nabla}_{r} \mathbf{v}$ of $n_{2}$. These are essentially arbitrary functions of $r_{12}$ and $t$; as we will see below, this prohibits direct calculation of the viscosities in the resulting Navier-Stokes-like equation. Inserting (33) into (32) and using that $\left[\int \mathrm{d} \mathbf{r} f(|\mathbf{r}|) \mathbf{r} \otimes \mathbf{r} /|\mathbf{r}|^{2}\right]_{\alpha, \beta}=1 / 3 \delta_{\alpha, \beta} \int \mathrm{d} \mathbf{r} f(|\mathbf{r}|)$ (where $[M]_{\alpha, \beta}$ denotes the $(\alpha, \beta)$ entry of the matrix $M$ ) along with (30) gives

$$
\begin{aligned}
\boldsymbol{\nabla}_{\mathbf{r}_{1}} \cdot \mathbf{P}^{(V)}= & \frac{1}{m} \boldsymbol{\nabla}_{\mathbf{r}_{1}} \cdot\left[\left(p^{(V)}-\zeta \boldsymbol{\nabla}_{\mathbf{r}_{1}} \cdot \mathbf{v}\right) \mathbf{1}\right. \\
& \left.-2 \eta^{(V)}\left(\boldsymbol{\Lambda}-\frac{1}{3} \mathbf{1} \boldsymbol{\nabla}_{\mathbf{r}_{1}} \cdot \mathbf{v}\right)\right]
\end{aligned}
$$

where

$$
\begin{aligned}
& p^{(V)}:=-\frac{1}{6} \int \mathrm{d} \mathbf{r}_{12}^{\prime} n_{2}^{(0)}\left(r_{12}^{\prime}, t\right) r_{12}^{\prime} \frac{\mathrm{d}}{\mathrm{d} r_{12}^{\prime}} V_{2}\left(r_{12}^{\prime}\right) \\
& \eta^{(V)}:=\frac{1}{30} \int \mathrm{d} \mathbf{r}_{12}^{\prime} B_{1}\left(r_{12}^{\prime}, t\right) r_{12}^{\prime} \frac{\mathrm{d}}{\mathrm{d} r_{12}^{\prime}} V_{2}\left(r_{12}^{\prime}\right) \\
& \zeta:=\frac{1}{6} \int \mathrm{d} \mathbf{r}_{12}^{\prime} B_{2}\left(r_{12}^{\prime}, t\right) r_{12}^{\prime} \frac{\mathrm{d}}{\mathrm{d} r_{12}^{\prime}} V_{2}\left(r_{12}^{\prime}\right) .
\end{aligned}
$$

We now define the pressure tensor to be (see (31) and (34)) $\mathbf{P}:=\frac{1}{m}\left[-2\left(\eta^{(K)}+\right.\right.$ $\left.\left.\eta^{(V)}\right)\left(\boldsymbol{\Lambda}-\frac{1}{3} \boldsymbol{\nabla}_{\mathbf{r}} \cdot \mathbf{v}\right)+p^{(V)} \mathbf{1}-\zeta \mathbf{1} \boldsymbol{\nabla}_{\mathbf{r}} \cdot \mathbf{v}\right]$. Using the identity $\boldsymbol{\nabla}_{\mathbf{r}} \cdot \boldsymbol{\Lambda}=\frac{1}{2} \Delta_{\mathbf{r}} \mathbf{v}+\frac{1}{2} \boldsymbol{\nabla}_{\mathbf{r}}\left(\boldsymbol{\nabla}_{\mathbf{r}} \cdot v\right)$ gives the out-of-equilibrium corrections as $\boldsymbol{\nabla}_{\mathbf{r}} \cdot \mathbf{P}=-\frac{1}{m} \eta \Delta_{\mathbf{r}} \mathbf{V}-\frac{1}{m}\left(\zeta+\frac{1}{3} \eta\right) \boldsymbol{\nabla}_{\mathbf{r}}\left(\boldsymbol{\nabla}_{\mathbf{r}} \cdot \mathbf{v}\right)$, where $\eta=\eta^{(K)}+\eta^{(V)}$.

Finally, we insert these corrections into (22), which along with (18) gives

$$
\begin{aligned}
m \rho\left(\mathbf{r}_{1}, t\right)\left(\partial_{t} \mathbf{v}\left(\mathbf{r}_{1}, t\right)+\mathbf{v}\left(\mathbf{r}_{1}, t\right) \cdot \boldsymbol{\nabla}_{\mathbf{r}} \mathbf{v}\left(\mathbf{r}_{1}, t\right)\right) \\
=\eta \Delta_{\mathbf{r}} \mathbf{v}\left(\mathbf{r}_{1}, t\right)+\left(\zeta+\frac{1}{3} \eta\right) \boldsymbol{\nabla}_{\mathbf{r}}\left(\boldsymbol{\nabla}_{\mathbf{r}} \cdot \mathbf{v}\left(\mathbf{r}_{1}, t\right)\right) \\
-\rho\left(\mathbf{r}_{1}, t\right) \boldsymbol{\nabla}_{\mathbf{r}_{1}} \frac{\delta \mathcal{F}[\rho]}{\delta \rho}-m \gamma \rho\left(\mathbf{r}_{1}, t\right) \mathbf{v}\left(\mathbf{r}_{1}, t\right) \\
-m \gamma \rho\left(\mathbf{r}_{1}, t\right) \int \mathrm{d} \mathbf{r}_{2}\left[\mathbf{Z}_{1}\left(\mathbf{r}_{1}, \mathbf{r}_{2}\right) \mathbf{v}\left(\mathbf{r}_{1}, t\right)+\mathbf{Z}_{2}\left(\mathbf{r}_{1}, \mathbf{r}_{2}\right) \mathbf{v}\left(\mathbf{r}_{2}, t\right)\right] \\
\times \rho\left(\mathbf{r}_{2}, t\right) g\left(\mathbf{r}_{1}, \mathbf{r}_{2},[\rho]\right) .
\end{aligned}
$$

This is a generalisation of the Navier-Stokes equation for a compressible colloidal fluid, which was stated in [42] for the case with no HI. It is worth reiterating Kreuzer's comment [56] that although we have formally derived a Navier-Stokes-like equation, the expressions for $p, \eta$ and $\zeta$ are not useful unless they can be evaluated explicitly. 
In addition, these quantities are constant in space as the system has been assumed to be isotropic; more realistic models would require dependence on $\rho$. In contrast to the Navier-Stokes equation, the above is an integro-differential equation for $\mathbf{v}$, non-local in both $\rho$ and $\mathbf{v}$. The effects of these non-localities, which contain the HI, are analogous to those discussed in Section 3.3. The term involving the free energy functional plays the role of the pressure and, at least under the square gradient approximation, it can be written as the divergence of a (Korteweg) stress tensor [71].

We note that the expansion in gradients of the velocity differs from the more standard expansion in gradients of the density in that it retains the full Helmholtz free energy functional, and thus, as in standard DFT, captures the correct microscopic structure. This is not true of expansions in the density, which leads to a mesoscopic theory.

An important addition to the equation obtained by going beyond the local equilibrium approximation is the introduction of the Laplacian of $\mathbf{v}$. This provides a dissipative, smoothing effect in the evolution, which is missing from (23). This is a common feature of theoretical models that fail to take certain effects (in this case the effective viscosity of the colloidal particles) into account. True physical systems should contain at least a small dissipative component, which smooths out any discontinuities. In contrast, approximate models can exhibit undesirable effects such as unphysical shocks or finite-time blow up.

\section{Reduction to dynamics of the density}

As discussed in the introduction, when $\gamma$ is large, the system is said to be overdamped and the evolution of the momentum becomes trivial. In this case, it can be shown rigorously [46] that the $N$-body Kramers equation reduces to the $N$-body Smoluchowski equation, depending only on the particle positions. An analogous result holds for the evolution of the one-body density [44], although the one-body diffusion tensor is now time-dependent. In this section we give a more heuristic derivation, similar in spirit to that of [42], but including HI and using some recent rigorous results [44].

As a first step, we assume that, in the high-friction limit, the local-equilibrium approximation

$$
f_{\mathrm{le}}^{(N)}\left(\mathbf{r}^{N}, \mathbf{p}^{N}, t\right)=\frac{\rho^{(N)}\left(\mathbf{r}^{N}, t\right)}{\left(2 \pi m k_{B} T\right)^{3 N / 2}} \prod_{i=1}^{N} \exp \left(-\frac{\left|\mathbf{p}_{i}-m \mathbf{v}\left(\mathbf{r}_{i}, t\right)\right|^{2}}{2 m k_{B} T}\right) .
$$

holds. Taking the divergence of (8), using the continuity equation (5) and its time derivative to replace the terms containing $\nabla_{\mathbf{r}_{1}} \cdot \mathbf{j}$ with time derivatives of the density, and inserting the local-equilibrium approximation gives

$$
\begin{array}{r}
\partial_{t}^{2} \rho\left(\mathbf{r}_{1}, t\right)+\gamma \partial_{t} \rho\left(\mathbf{r}_{1}, t\right)-\nabla_{\mathbf{r}_{1}} \cdot \mathbf{A}_{\mathrm{le}}\left(\mathbf{r}_{1}, t\right) \\
=\frac{1}{m} \nabla_{\mathbf{r}_{1}} \cdot\left(\rho\left(\mathbf{r}_{1}, t\right) \boldsymbol{\nabla}_{\mathbf{r}_{1}} \frac{\delta \mathcal{F}[\rho]}{\delta \rho}\right)
\end{array}
$$




$$
+\gamma N \nabla_{\mathbf{r}_{1}} \cdot \sum_{j=1}^{N} \int \mathrm{d} \mathbf{r}^{N-1} \tilde{\boldsymbol{\Gamma}}_{1 j}\left(\mathbf{r}^{N}\right) \mathbf{v}\left(\mathbf{r}_{j}, t\right) \rho^{(N)}\left(\mathbf{r}^{N}, t\right),
$$

where $\mathbf{A}_{\mathrm{le}}$ is given by (17) with $f_{\text {neq }}=0$. If we were to now neglect HI and make a local equilibrium assumption, we would recover the standard DDFT [21] with the addition of a second-order time derivative. Whilst this term can be heuristically neglected in the high-friction limit, its effect could be important when interest lies in the microscopic dynamics, i.e. on short timescales. Physically, this term introduces a finite propagation speed, which can also be derived by including memory effects [72]. For the present analysis, we will restrict our attention to systems in which the second derivative is negligible in order to compare with existing DDFTs.

To obtain a closed equation for $\rho$, it remains to treat the term $\boldsymbol{\nabla}_{\mathbf{r}_{1}} \cdot \mathbf{A}_{\text {le }}$ and the HI terms. We first use the results of [44], which show that, at least for two-body potential and friction terms and under the Enskog approximation, terms containing $\mathbf{A}$ are negligible in the high-friction limit. At this point we have recovered the corresponding result of [42], but using the rigorous results of [44]. To treat the HI terms, is necessary to find a closure relation, expressing $\mathbf{v}$ as a functional of $\rho$.

To do so, we assume that the material derivative of the velocity is negligible, i.e. that the velocity changes only due to transport of the flow, and (8) becomes

$$
\begin{aligned}
\frac{1}{m} \rho\left(\mathbf{r}_{1}, t\right) \boldsymbol{\nabla}_{\mathbf{r}_{1}} & \frac{\delta \mathcal{F}[\rho]}{\delta \rho}+\gamma \rho\left(\mathbf{r}_{1}, t\right) \mathbf{v}\left(\mathbf{r}_{1}, t\right) \\
& +\gamma N \sum_{j=1}^{N} \int \mathrm{d} \mathbf{r}^{N-1} \tilde{\boldsymbol{\Gamma}}_{1 j}\left(\mathbf{r}^{N}\right) \mathbf{v}\left(\mathbf{r}_{j}, t\right) \rho^{(N)}\left(\mathbf{r}^{N}, t\right)=0 .
\end{aligned}
$$

Since $\rho^{(N)}$ is a functional of $\rho,(37)$ in principle determines $\mathbf{v}$ as a functional of $\rho$. However, this functional is in no way explicit and we cannot make a simple connection to the Smoluchowski limit. We now consider two cases in which we can treat this issue. The first demonstrates a difference in the definition of the diffusion tensor (which is also present in the rigorous derivation [44]), whilst the second recovers the result of [25] under an additional assumption.

\section{1. $\Gamma$ is $3 \times 3$ block diagonal}

We assume that $\boldsymbol{\Gamma}$ is block diagonal with $3 \times 3$ blocks, i.e. $\tilde{\boldsymbol{\Gamma}}_{i j}=0$ for $i \neq j$. Whilst for unconfined systems studied here, the leading order terms occur in $\tilde{\boldsymbol{\Gamma}}_{i j}, i \neq j$, for confined systems in which the particles are well-separated but close to walls, the diagonal blocks $\tilde{\boldsymbol{\Gamma}}_{i i}$ are expected to dominate [73]. In addition, this regime allows us to more clearly demonstrate the connection between $\boldsymbol{\Gamma}$ and $\mathbf{D}$ by allowing an explicit solution of (37).

Setting $\tilde{\boldsymbol{\Gamma}}_{i j}=0$ for $i \neq j$ in (37) and using the identity $\int \mathrm{d} \mathbf{r}^{N-1} \rho^{(N)}\left(\mathbf{r}^{N}, t\right)=$ $\rho\left(\mathbf{r}_{1}, t\right) / N$ gives

$$
\gamma \mathbf{v}\left(\mathbf{r}_{1}, t\right)=-\frac{\rho(\mathbf{r}, t)}{m N}\left(\int \mathrm{d} \mathbf{r}^{N-1} \boldsymbol{\Gamma}_{11}\left(\mathbf{r}^{N}\right) \rho^{(N)}\left(\mathbf{r}^{N}, t\right)\right)^{-1} \nabla_{\mathbf{r}_{1}} \frac{\delta \mathcal{F}[\rho]}{\delta \rho},
$$


where we have recalled the definition $\boldsymbol{\Gamma}_{11}=\mathbf{1}+\tilde{\boldsymbol{\Gamma}}_{11}$. Since $\boldsymbol{\Gamma}_{11}$ is a principal minor of a positive definite matrix, it is also positive definite. Using that $\rho^{(N)} \geq 0$ and not zero everywhere shows that, for any non-zero $\mathbf{w}\left(\mathbf{r}_{1}\right)$,

$$
\begin{aligned}
\mathbf{w}\left(\mathbf{r}_{1}\right) \cdot\left[\int \mathrm{d} \mathbf{r}^{N-1} \boldsymbol{\Gamma}_{11}\left(\mathbf{r}^{N}\right) \rho^{(N)}\left(\mathbf{r}^{N}, t\right)\right] \mathbf{w}\left(\mathbf{r}_{1}\right) \\
=\int \mathrm{d} \mathbf{r}^{N-1} \mathbf{w}\left(\mathbf{r}_{1}\right) \cdot \boldsymbol{\Gamma}_{11}\left(\mathbf{r}^{N}\right) \mathbf{w}\left(\mathbf{r}_{1}\right) \rho^{(N)}\left(\mathbf{r}^{N}, t\right)>0,
\end{aligned}
$$

and the integral in square brackets is a positive definite matrix and thus invertible on functions of $\mathbf{r}_{1}$.

For ease of notation, we define $\widehat{\mathbf{M}}\left(\mathbf{r}_{1}, t\right)=\int \mathrm{d} \mathbf{r}^{N-1} \mathbf{M}\left(\mathbf{r}^{N}\right) \rho^{(N)}\left(\mathbf{r}^{N}, t\right)$. Recalling $\tilde{\boldsymbol{\Gamma}}_{i j}=0$ for $i \neq j$ and using (38) to replace $\mathbf{v}\left(\mathbf{r}_{1}, t\right)$ in the final term of (36), which can be taken out of the integral, gives

$\partial_{t}^{2} \rho\left(\mathbf{r}_{1}, t\right)+\gamma \partial_{t} \rho\left(\mathbf{r}_{1}, t\right)-\nabla_{\mathbf{r}_{1}} \cdot \mathbf{A}_{\mathrm{le}}\left(\mathbf{r}_{1}, t\right)$

$=\frac{1}{m} \boldsymbol{\nabla}_{\mathbf{r}_{1}} \cdot\left(\rho\left(\mathbf{r}_{1}, t\right) \mathbf{1}-N \widehat{\mathbf{1}}\left(\mathbf{r}_{1}, t\right) \widehat{\tilde{\boldsymbol{\Gamma}}}_{11}\left(\mathbf{r}_{1}, t\right) \widehat{\boldsymbol{\Gamma}}_{11}^{-1}\left(\mathbf{r}_{1}, t\right)\right) \boldsymbol{\nabla}_{\mathbf{r}_{1}} \frac{\delta \mathcal{F}[\rho]}{\delta \rho}$

We now demonstrate the connection to the generalization of $[25,(14)]$, with $\omega_{11}=\tilde{\mathbf{D}}_{11}$ and $\omega_{12}=0$, i.e.

$$
\frac{k_{B} T}{D_{0}} \partial_{t} \rho\left(\mathbf{r}_{1}, t\right)=\nabla_{\mathbf{r}_{1}} \cdot\left(\rho\left(\mathbf{r}_{1}, t\right) \mathbf{1}+N \widehat{\tilde{\mathbf{D}}_{11}}\left(\mathbf{r}_{1}, t\right)\right) \boldsymbol{\nabla}_{\mathbf{r}_{1}} \frac{\delta \mathcal{F}[\rho]}{\delta \rho} .
$$

We assume that in the large- $\gamma$ limit, inertia effects are negligible, and thus may ignore the $\partial_{t}^{2} \rho$ term. Using the standard definition $D_{0}=k_{B} T /(m \gamma)$, we find

$$
\widehat{\tilde{\mathbf{D}}_{11}}\left(\mathbf{r}_{1}, t\right)=-\widehat{\mathbf{1}}\left(\mathbf{r}_{1}, t\right) \widehat{\tilde{\boldsymbol{\Gamma}}}_{11}\left(\mathbf{r}_{1}, t\right) \widehat{\boldsymbol{\Gamma}}_{11}^{-1}\left(\mathbf{r}_{1}, t\right) \text {. }
$$

Using the standard definition $\mathbf{D}=k_{B} T / m \boldsymbol{\Gamma}^{-1}$, would give $\mathbf{D}=D_{0}(\mathbf{1}+\tilde{\mathbf{D}})$ with $\tilde{\mathbf{D}}_{i i}=-\mathbf{1} \tilde{\boldsymbol{\Gamma}}_{i i}\left(\boldsymbol{\Gamma}_{i i}\right)^{-1}$ (where we have kept $\mathbf{1}$ to mirror (40)) and $\mathbf{D}_{i j}=0$ for $i \neq j$. Hence, (40) is reminiscent of the the standard formulation, except each of the terms must be averaged against $\rho^{N}$ over all but one spatial coordinate.

It is noteworthy that the novel form of the relationship (40) result from the noncommutativity of the two processes of integrating over $N-1$ particles and taking the large- $\gamma$, see [44] for further discussions. If we repeat the analysis described above, but do not integrate over $\mathbf{r}^{N-1}$ until after taking the large- $\gamma$ limit, we obtain the standard definition of the diffusion tensor. It would be interesting to investigate the differences between these two formulations.

\subsection{Existence of a small parameter}

As discussed previously, assuming that $\mathbf{D}$ contains only two-body terms is not equivalent to assuming the same of $\boldsymbol{\Gamma}$. In this section we wish to investigate what happens when the $\tilde{\boldsymbol{\Gamma}}_{i j}$ 's are of the form $\epsilon \tilde{\boldsymbol{\Gamma}}_{i j}^{\prime}$, where $\tilde{\boldsymbol{\Gamma}}_{i j}^{\prime}$ is of $O(1)$ and $\epsilon$ is a small parameter (independent of $\gamma, N, \mathbf{r}$ ) such that $\tilde{\boldsymbol{\Gamma}}_{i j} \tilde{\boldsymbol{\Gamma}}_{k \ell}$ is negligible when compared to $\tilde{\boldsymbol{\Gamma}}_{i j}$. We note that this is equivalent to $\tilde{\mathbf{D}}_{i j}$ being of the form $\epsilon \tilde{\mathbf{D}}_{i j}^{\prime}$ with the same assumptions. This can be seen by setting $\boldsymbol{\Gamma}=\gamma(\mathbf{1}+\tilde{\boldsymbol{\Gamma}})$ and $\mathbf{D}=D_{0}(\mathbf{1}+\tilde{\mathbf{D}})$, we have $\tilde{\mathbf{D}}=-\tilde{\boldsymbol{\Gamma}}(\mathbf{1}+\tilde{\boldsymbol{\Gamma}})^{-1}=$ 
$-\tilde{\boldsymbol{\Gamma}} \sum_{n=0}^{\infty}(-\tilde{\boldsymbol{\Gamma}})^{n} \approx-\tilde{\boldsymbol{\Gamma}}$. Hence, by symmetry of the argument, the $\tilde{\boldsymbol{\Gamma}}_{i j}^{n}$ for $n \geq 2$ are negligible if and only if the $\tilde{\mathbf{D}}_{i j}^{n}$ are negligible. An example is the Rotne-Prager (27) two-body expansion (26) with the assumption that the interparticle distances are large compared to $\sigma_{\mathrm{H}}$, for example in a dilute fluid where the repulsive forces are long range compared to $\sigma_{\mathrm{H}}$. Then $\sigma_{\mathrm{H}} /\left|\mathbf{r}-\mathbf{r}^{\prime}\right|$ is a small parameter.

From (37) we see that $\gamma \tilde{\boldsymbol{\Gamma}}_{1 j} \mathbf{v}\left(\mathbf{r}_{j}, t\right)=-\tilde{\boldsymbol{\Gamma}}_{1 j} \boldsymbol{\nabla}_{\mathbf{r}_{j}} \frac{\delta \mathcal{F}[\rho]}{\delta \rho}+\mathcal{O}\left(\epsilon^{2}\right)$ and hence, up to errors of $\mathcal{O}\left(\epsilon^{2}\right)$, (36) becomes,

$$
\begin{aligned}
\gamma \partial_{t} \rho\left(\mathbf{r}_{1}, t\right)= & \frac{1}{m} \boldsymbol{\nabla}_{\mathbf{r}_{1}} \cdot\left(\rho\left(\mathbf{r}_{1}, t\right) \boldsymbol{\nabla}_{\mathbf{r}_{1}} \frac{\delta \mathcal{F}[\rho]}{\delta \rho}\right) \\
& -\frac{N}{m} \nabla_{\mathbf{r}_{1}} \cdot \sum_{j=1}^{N} \int \mathrm{d} \mathbf{r}^{N-1} \tilde{\boldsymbol{\Gamma}}_{1 j}\left(\mathbf{r}^{N}\right) \boldsymbol{\nabla}_{\mathbf{r}_{j}} \frac{\delta \mathcal{F}[\rho]}{\delta \rho\left(\mathbf{r}_{j}, t\right)} \rho^{(N)}\left(\mathbf{r}^{N}, t\right),
\end{aligned}
$$

where we have once again chosen to neglect the $\partial_{t}^{2} \rho$ and $\boldsymbol{\nabla} \cdot \mathbf{A}$ terms. Using $\tilde{\boldsymbol{\Gamma}}_{i j}=-\tilde{\mathbf{D}}_{i j}$ recovers the DDFT previously derived by Rex and Löwen [25]. As these authors discussed, and as with the velocity equation (23) (see end of Section 3.3), it is necessary to close this equation by determining $\rho^{(2)}$, or equivalently $g$, in terms of $\rho$. When HI are neglected by setting $\tilde{\mathbf{D}}_{i j}=0$, this DDFT reduces to that of Marconi and Tarazona [21].

\section{Discussion and Open Problems}

We have derived a general DDFT (equations (5) and (23)) for systems of colloidal particles, including the effects of inertia and hydrodynamic interactions. The derivation requires three approximations, (i) the adiabatic approximation that the $n$-body correlations are equal to those of an equilibrium system with the same one-body distribution and we thus employ the sum rule (7), which holds exactly in equilibrium; (ii) that the contributions from the part of the one-body distribution which is not captured by the local-equilibrium approximation may be neglected or approximated by a known functional of $\rho$ and $\mathbf{v}$; and (iii) that the $n$-body distributions governing the HI terms may be well-approximated by functionals of $\rho$ and $\mathbf{v}$. For two-body HI, it was demonstrated that the widely-used Enskog approximation is sufficient to obtain accurate dynamics. By neglecting HI we recover the DDFT derived by Archer [42].

We have demonstrated the very good quantitative and qualitative agreement between the DDFT and the full underlying Langevin dynamics for both 1D hard rod and 3D hard sphere systems. In both cases, the inclusion of both inertia and HI is crucial in obtaining the correct dynamics. We further demonstrated the advantages of the DDFT by computing the dynamics of 1000 particles, the Langevin calculations for which are prohibitively computationally expensive.

Close to local-equilibrium, we obtain a generalized Navier-Stokes-like equation. This integro-differential equation contains non-local terms describing HI effects. In the high friction limit we recover the DDFTs of Marconi and Tarazona [21] and Rex and Löwen [25]. However, in the latter case, unless we make an additional assumption on the form of the HI, we obtain a different definition of the diffusion tensor, the effects 
of which are worth further study.

There are many promising extensions to the theory formulated here including the extension to multiple particle species, anisotropic particles, self-propelled particles, confined geometries, and the inclusion of an external flow. Such extensions would allow the study of many systems of physical interest including the delivery of drug-laden nanoparticles in the circulatory system, cloud formation and wetting phenomena. As discussed in the Introduction, it is expected that both inertia and HI play important roles in the dynamics of these and many other related systems. In addition, we plan to extend our numerical implementation of the various DDFT formalisms to two dimensions, allowing the study of the effects of inertia and HI in a much wider range of systems, such as the HI-induced symmetry breaking of driven colloidal particles [74].

\section{Acknowledgements}

We are grateful to the European Research Council via Advanced Grant No. 247031, the Rotary Clubs Darmstadt, Darmstadt-Bergstrasse and Darmstadt-Kranichstein, the European Framework 7 via Grant No. 214919 (Multiflow) and the Engineering and Physical Sciences Research Council of the UK via grant No. EP/H034587/ for support of this research.

[1] J. K. G. Dhont. An Introduction to Dynamics of Colloids. Elsevier, 1996.

[2] R. Brown. A brief account of microscopical observations made on the particles contained in the pollen of plants. Phil. Mag., 4:161-173, 1828.

[3] A. Einstein. The presumed movement of suspended particles in static fluids. Ann. Phys. Lpz, 17:549-560, 1905.

[4] P. Langevin. Sur la théorie du mouvement brownien. C. R. Acad. Sci. (Paris), 146:530-533, 1908.

[5] M. Von Smoluchowski. Uber Brownische Molekularbewegung unter Einwirkung äusserer Kräfte und deren Zusammenhang mit der verallgemeinerten diffusionsgleichung. Ann. Phys, 48:1103$1112,1915$.

[6] J. Perrin. Mouvement Brownien er réalité moléculaire. Ann. Chim. Phys., 18:1-114, 1909.

[7] T. Franosch, M. Grimm, M. Belushkin, F.M. Mor, G. Foffi, L. Forró, and S. Jeney. Resonances arising from hydrodynamic memory in Brownian motion. Nature, 478(7367):85-88, 2011.

[8] R. Huang, I. Chavez, K.M. Taute, B. Lukić, S. Jeney, M. G. Raizen, and E. L. Florin. Direct observation of the full transition from ballistic to diffusive Brownian motion in a liquid. Nature Physics, 7(7):576-580, 2011.

[9] E. Matijević, editor. Medical Applications of Colloids. Springer, New York, 2008.

[10] D. F. Evans and H. Wennerström. The Colloidal Domain: Where Physics, Chemistry, and Biology Meet. Wiley, New York, 1999.

[11] H.A. Stone and S. Kim. Microfluidics: Basic issues, applications and challenges. AIChE J., 47:1250-1254, 2001.

[12] F. Caruso. Colloids and Colloid Assemblies: Synthesis, Modification, Organization and Utilization of Colloid Particles. Wiley, Weinheim, 2004.

[13] G. K.-L. Chan and R. Finken. Time-dependent density functional theory of classical fluids. Phys. Rev. Lett., 94:183001, 2005.

[14] R. Evans. The nature of the liquid-vapour interface and other topics in the statistical mechanics of non-uniform, classical fluids. Adv. Phys., 28(2):143, 1979. 
[15] E. Roman and W. Dieterich. Classical fluid in a periodic potential and the density-functional approach. Phys. Rev. A, 32(6):3726, 1985.

[16] J.-Z. Wu. Density functional theory for chemical engineering: From capillarity to soft materials. AIChE J., 52:1169-1193, 2006.

[17] J.-Z. Wu and Z.-D. Li. Density-functional theory for complex fluids. Annu. Rev. Phys. Chem., $58: 85-112,2007$.

[18] G. Nägele. On the dynamics and structure of charge-stabilized suspensions. Phys. Rep., 272:215$372,1996$.

[19] J. T. Padding and A. A. Louis. Hydrodynamic interactions and Brownian forces in colloidal suspensions: Coarse-graining over time and length scales. Phys. Rev. E, 74:031402, 2006.

[20] W. Dietrich, H. L. Frisch, and A. Majhofer. Nonlinear diffusion and density functional theory. Z. Phys. B, 78:317, 1990.

[21] U. M. B. Marconi and P. Tarazona. Dynamic density functional theory of fluids. J. Chem. Phys., 110(16):8032, 1999.

[22] U. M. B. Marconi and P. Tarazona. Dynamic density functional theory of fluids. J. Phys. Condens. Matter, 12:A413, 2000.

[23] A. J. Archer and R. Evans. Dynamical density functional theory and its application to spinodal decomposition. J. Chem. Phys., 121(9):4246, 2004.

[24] P. Español and H. Löwen. Derivation of dynamical density functional theory using the projection operator technique. J. Chem. Phys., 131:244101, 2009.

[25] M. Rex and H. Löwen. Dynamical density functional theory for colloidal dispersions including hydrodynamic interactions. Eur. Phys. J. E, 28:139, 2009.

[26] C.P. Royall, J. Dzubiella, M. Schmidt, and A. Van Blaaderen. Nonequilibrium sedimentation of colloids on the particle scale. Phys. Rev. Lett., 98(18):188304, 2007.

[27] M. Rauscher. DDFT for Brownian particles and hydrodynamics. J. Phys. Condens. Matter, 22:364109, 2010.

[28] P. Yatsyshin, N. Savva, and S. Kalliadasis. Spectral methods for the equations of classical densityfunctional theory: Relaxational dynamics of microscopic films. J. Chem. Phys., 136:124113, 2012.

[29] A. Pereira and S. Kalliadasis. Equilibrium gas-liquid-solid contact angle from density-functional theory. J. Fluid Mech., 692:53-77, 2012.

[30] N. Savva, S. Kalliadasis, and G.A. Pavliotis. Two-dimensional droplet spreading over random topographical substrates. Phys. Rev. Lett., 104:084501, 2010.

[31] E. Gavze and M. Shapiro. Motion of inertial spheroidal particles in a shear flow near a solid wall with special application to aerosol transport in microgravity. J. Fluid Mech., 371(1):59-79, 1998.

[32] H. R. Pruppacher, J. D. Klett, and P. K. Wang. Microphysics of clouds and precipitation. Taylor \& Francis, 1998.

[33] H. Sigurgeirsson and A. M. Stuart. A model for preferential concentration. Phys. Fluids, 14(12):4352-4361, 2002.

[34] G. Falkovich, A. Fouxon, and M.G. Stepanov. Acceleration of rain initiation by cloud turbulence. Nature, 419:151-154, 2002.

[35] P. Worth Longest and J. Xi. Computational investigation of particle inertia effects on submicron aerosol deposition in the respiratory tract. J. Aerosol Sci., 38(1):111-130, 2007.

[36] P. Worth Longest and C. Kleinstreuer. Comparison of blood particle deposition models for nonparallel flow domains. J. Biomech., 36(3):421 - 430, 2003.

[37] U. M. B. Marconi and S. Melchionna. Phase-space approach to dynamical density functional theory. J. Chem. Phys., 126:184109, 2007.

[38] U. M. B. Marconi, P. Tarazona, and F. Cecconi. Theory of thermostatted inhomogeneous granular fluids: A self-consistent density functional description. J. Chem. Phys., 126:164904, 2007.

[39] U. M. B. Marconi and P. Tarazona. Nonequilibrium inertial dynamics of colloidal systems. $J$. 
Chem. Phys., 124:164901, 2006.

[40] P. Tarazona and U. M. B. Marconi. Beyond the dynamic density functional theory for steady currents: Application to driven colloidal particles in a channel. J. Chem. Phys., 128:164704, 2008.

[41] U. M. B. Marconi, P. Tarazona, F. Cecconi, and S. Melchionna. Beyond dynamic density functional theory: the role of inertia. J. Phys. Condens. Matter, 20:494233, 2008.

[42] A. J. Archer. Dynamical density functional theory for molecular and colloidal fluids: A microscopic approach to fluid mechanics. J. Chem. Phys., 130(1):014509, 2009.

[43] B. D. Goddard, A. Nold, N. Savva, G. A. Pavliotis, and S. Kalliadasis. General dynamical density functional theory for classical fluids. Phys. Rev. Lett., 109:120603, 2012.

[44] B. D. Goddard, G. A. Pavliotis, and S. Kalliadasis. The overdamped limit of dynamical density functional theory: Rigorous results. SIAM Multiscale Model. Sim., 10:633-663, 2012.

[45] D. L. Ermak and J. A. McCammon. Brownian dynamics with hydrodynamic interactions. J. Chem. Phys., 69(4):1351, 1978.

[46] U. M. Titulaer. Corrections to the Smoluchowski equation in the presence of hydrodynamic interactions. Physica A, 100:251, 1980.

[47] E. Runge and E. K. U. Gross. Density-functional theory for time-dependent systems. Phys. Rev. Lett., 52:997-1000, Mar 1984.

[48] J. K. Percus. Equilibrium state of a classical fluid of hard rods in an external field. J. Stat. Phys., 15(6):505-511, 1976.

[49] Y. Rosenfeld. Free-energy model for the inhomogeneous hard-sphere fluid mixture and densityfunctional theory of freezing. Phys. Rev. Lett., 63(9):980-983, 1989.

[50] Y. Rosenfeld, M. Schmidt, H. Löwen, and P. Tarazona. Fundamental-measure free-energy density functional for hard spheres: Dimensional crossover and freezing. Phys. Rev. E, 55(4):4245, 1997.

[51] R. Roth, R. Evans, A. Lang, and G. Kahl. Fundamental measure theory for hard-sphere mixtures revisited: the White Bear version. J. Phys. Condens. Matter, 14:12063, 2002.

[52] R. Roth. Fundamental measure theory for hard-sphere mixtures: a review. Journal of Physics: Condensed Matter, 22:063102, 2010.

[53] A. J. Archer. Dynamical density functional theory: binary phase-separating colloidal fluid in a cavity. J. Phys. Condens. Matter, 17:1405, 2005.

[54] J. F. Lutsko. Recent developments in classical density functional theory. Adv. Chem. Phys., 144:1, 2010.

[55] C. N. Likos, A. Lang, M. Watzlawek, and H. Löwen. Criterion for determining clustering versus reentrant melting behavior for bounded interaction potentials. Phy. Rev. E, 63(3):031206, 2001.

[56] H. J. Kreuzer. Noneqilibrium Thermodynamics and Its Statistical Foundations. oxford University Press, New York, 1981.

[57] S. Succi. The lattice Boltzmann equation for fluid dynamics and beyond. Oxford Scientific Publications, 2001.

[58] D. Jou, J. Casas-Vázquez, and G. Lebon. Exetended Irreversible Thermodynamics. Springer Verlag Heidelberg, 1996.

[59] S. Chapman and Cowling T. G. The mathematical theory of non-uniform gases: an account of the kinetic theory of viscosity, thermal conduction and diffusion in gases. Cambridge University Press, 1990.

[60] C. F. Dunkl and Y. Xu. Orthogonal Polynomials of Several Variables. Cambridge University Press, 2001.

[61] D. J. Jeffrey and Y. Onishi. Calculation of the resistance and mobility functions for two unequal rigid spheres in low-reynolds-number flow. Journal of Fluid Mechanics, 139(1):261-290, 1984.

[62] H. A. Knudsen, J. H. Werth, and D. E. Wolf. Failure and success of hydrodynamic interaction models. The European Physical Journal E: Soft Matter and Biological Physics, 27(2):161-170, 2008.

[63] S. G. Brush. Kinetic theory, Vol.3, The Chapman-Enskog solution of the transport equation for 
moderately dense gases. Pergamon, London, 1972.

[64] H. Van Beijeren and M. H. Ernst. The modified Enskog equation. Physica, 68(3):437-456, 1973.

[65] P. Résibois and M. De Leener. Classical Kinetic Theory of Fluids. Wiley, 1977.

[66] P. E. Kloeden and E. Platen. Numerical Solution of Stochastic Differential Equations. SpringerVerlag, Berlin, 1992.

[67] J. M. Brader. Nonlinear rheology of colloidal dispersions. Journal of Physics: Condensed Matter, 22:363101, 2010.

[68] J. P. Boyd. Chebyshev and Fourier Spectral Methods. Dover, UK, 2001.

[69] E. Hairer and G. Wanner. Solving Ordinary Differential Equations II. Stiff and DifferentialAlgebraic Problems, volume 14 of Springer Series in Computational Mathematics. SpringerVerlag, Berlin, 2006.

[70] J. H. Irving and J. G. Kirkwood. The statistical mechanical theory of transport processes. IV. The equations of hydrodynamics. J. Chem. Phys., 18(6):817, 1950.

[71] W. Mickel, L. Joly, and T. Biben. Transport, phase transitions, and wetting in micro/nanochannels: A phase field/ddft approach. J. Chem. Phys, 134:094105, 2011.

[72] T. Koide, G. Krein, and R. O. Ramos. Incorporating memory effects in phase separation processes. Physics Letters B, 636(2):96-100, 2006.

[73] J. R. Blake. A note on the image system for a stokeslet in a no-slip boundary. Proc. Camb. Phil. Soc, 70:303-310, 1971.

[74] Y. Sokolov, D. Frydel, D.G. Grier, H. Diamant, and Y. Roichman. Hydrodynamic pair attractions between driven colloidal particles. Physical Review Letters, 107(15):158302, 2011. 